The Astrophysical Journal, 613:1270-1284, 2004 October 1

(C) 2004. The American Astronomical Society. All rights reserved. Printed in U.S.A.

\title{
SEARCH FOR NANOSECOND OPTICAL PULSES FROM NEARBY SOLAR-TYPE STARS
}

\author{
Andrew W. Howard, ${ }^{1}$ Paul Horowitz, ${ }^{1}$ David T. Wilkinson, ${ }^{2,3}$ Charles M. Coldwell, ${ }^{1}$ Edward J. Groth, ${ }^{2}$ Norm JarosiK, ${ }^{2}$ \\ David W. Latham, ${ }^{4}$ Robert P. Stefanik, ${ }^{4}$ Alexander J. Willman, JR., ${ }^{5}$ Jonathan Wolff, ${ }^{1}$ and Joseph M. Zajac ${ }^{4}$ \\ Received 2004 March 16; accepted 2004 June 11
}

\begin{abstract}
With "Earth 2000" technology we could generate a directed laser pulse that outshines the broadband visible light of the Sun by 4 orders of magnitude. This is a conservative lower bound for the technical capability of a communicating civilization; optical interstellar communication is thus technically plausible. We have built a pair of systems to detect nanosecond pulsed optical signals from a target list that includes some 13,000 Sun-like stars, and we have made some 16,000 observations totaling nearly $2400 \mathrm{hr}$ during five years of operation. A beam splitter-fed pair of hybrid avalanche photodetectors at the $1.5 \mathrm{~m}$ Wyeth Telescope at the Harvard/Smithsonian Oak Ridge Observatory (Agassiz Station) triggers on a coincident pulse pair, initiating measurement of pulse width and intensity at subnanosecond resolution. An identical system at the $0.9 \mathrm{~m}$ Cassegrain at Princeton's Fitz-Randolph Observatory performs synchronized observations with $0.1 \mu$ s event timing, permitting unambiguous identification of even a solitary pulse. Among the 11,600 artifact-free observations at Harvard, the distribution of 274 observed events shows no pattern of repetition, and is consistent with a model with uniform event rate, independent of target. With one possible exception (HIP 107395), no valid event has been seen simultaneously at the two observatories. We describe the search and candidate events and set limits on the prevalence of civilizations transmitting intense optical pulses.
\end{abstract}

Subject headings: astrobiology — extraterrestrial intelligence — instrumentation: detectors techniques: photometric

\section{INTRODUCTION}

Historically the Cocconi \& Morrison (1959) suggestion that the search for extraterrestrial intelligence (SETI) be carried out at the $21 \mathrm{~cm}$ emission wavelength of neutral hydrogen came at a time in our technological development when no other astronomical lines were known in the microwave and there were no operational lasers. The rapid development of laser technology since that time - a Moore's law doubling of capability roughly every year-along with the discovery of many microwave lines of astronomical interest, have lessened somewhat the allure of hydrogen-line SETI. Indeed, on Earth the exploitation of photonics has revolutionized communications technology, with high-capacity fibers replacing both the historical copper cables and the long-haul microwave repeater chains. In addition, the elucidation (Cordes \& Lazio 1991) of the consequences to SETI of interstellar dispersion (first seen in pulsar observations) has broadened thinking about optimum wavelengths. Even operating under the prevailing criterion of minimum energy per bit transmitted, one is driven upward to millimetric wavelengths.

Moreover, there are other considerations that might well encourage the use of still shorter wavelengths. A transmitting

\footnotetext{
1 Physics Department, Harvard University, Cambridge, MA 02138; howard@ physics.harvard.edu, horowitz@physics.harvard.edu, coldwell@physics.harvard .edu, jwolff@physics.harvard.edu.

2 Physics Department, Princeton University, Princeton, NJ 08544; groth@ pupgg.princeton.edu, jarosik@pupgg.princeton.edu.

3 Deceased; initiated the Princeton project, but not responsible for errors in this paper.

4 Harvard-Smithsonian Center for Astrophysics, 60 Garden Street, Cambridge, MA 02138; dlatham@cfa.harvard.edu, rstefanik@cfa.harvard .edu, jzajac@cfa.harvard.edu.

5 Office of Information Technology, Princeton University, Princeton, NJ 08544; willman@princeton.edu.
}

civilization might wish to minimize transmitter size or weight, or use a system capable of great bandwidth, or perhaps design a beacon that is very easy to detect.

In comparing the relative merits of radio versus optical, it has sometimes been incorrectly assumed that one would always prefer coherent (heterodyne) detection and that the noise background is given by an effective temperature $T_{n}=h \nu / k$. For ultrahigh-resolution spectroscopy one must use such a system, mixing the optical signal down to microwave frequencies where radio techniques can be used, but if one is interested instead in the detection of short pulses, it is far better to use photon-counting detectors (e.g., photomultipliers; Ross 1965). That is because the process of heterodyning and linear detection is intrinsically noisy, for fundamental reasons: because heterodyne detection allows a measurement of phase, there must be uncertainty in the amplitude. The added noise is immaterial in the radio region, where there are many photons per mode, but it is serious in the optical, where the photon field is dilute.

Taking these and other factors into account in a comparison of received signal-to-noise ratio $(\mathrm{S} / \mathrm{N})$ versus wavelength and making reasonable assumptions about antenna apertures and accuracies, detection methods, transmitter power, and so on, Townes (1983) concluded that optical methods are comparable, or perhaps slightly preferred, in the single figure of merit of delivered $\mathrm{S} / \mathrm{N}$ for a given transmitter power. Other factors are obviously important - for example, penetration of an atmosphere (which favors microwave) or high data rates (which favors optical) - and could easily tip the balance. The conclusion is that the SETI community's historical bias toward microwaves should be reconsidered (Schwartz \& Townes 1961).

Laser technology has been in a phase of rapid catch-up relative to the mature technology at radio frequencies. Lasers with several megawatts of continuous optical output have been 
built, and picosecond pulses of more than a petawatt $\left(10^{15} \mathrm{~W}\right)$ have been produced. Progress in solid-state lasers has been impressive, and there are laser designs on the drawing board to produce repetitively pulsed megajoule nanosecond pulses. Optical pulsed beacons formed with that sort of technology permit detection with a very simple apparatus - just a telescope with a pair of white-light photomultipliers in coincidence.

\subsection{Pulses versus Carriers}

Are pulses the best beacon? Or should we be looking for laser lines, transmitted continuously at some guessable wavelength, analogous to the microwave searches that have been conducted?

What is natural at radio frequencies may not be so at optical. At radio frequencies it is easy to do coherent detection, using the ordinary heterodyne techniques of mixing with a local oscillator to a complex (quadrature) baseband. With classical filter techniques, or with contemporary digital processing with discrete Fourier transforms, one can achieve extremely narrow bandwidths, limited only by oscillator stability (a part in $10^{9}$ is routine) and patience (the resolution is the inverse of the coherent integration time). Furthermore, the interstellar medium is kind to carriers - at gigahertz frequencies a carrier is broadened only millihertz in its passage through the interstellar medium, if one avoids the most congested region of the Galactic center, and even there the broadening is only a few hertz (Cordes \& Lazio 1991). Scattering and absorption are also small or negligible over Galactic distances for such signals. In other words, a signal that is a spike in the frequency domain is a natural candidate for interstellar signaling at microwave frequencies, for reasons both scientific and technical.

Moreover, interstellar dispersion and the presence of natural and "cultural" impulsive interference (switching transients, spark plugs, and so on) make pulses in time less effective. Finally, the relatively low carrier frequency (along with dispersion) prevents high-bandwidth communications.

By contrast, at optical wavelengths the situation is reversed: one cannot realize extremely narrowband systems with optical filters or gratings, but is forced to optical heterodyne techniques, ultimately applying precise radio-frequency spectroscopic methods at the microwave intermediate frequency. This results in added noise, as mentioned above and well described by Townes (1983). Furthermore, at optical wavelengths the higher carrier frequencies $\left(\sim 10^{14} \mathrm{~Hz}\right)$ result in much larger absolute Doppler shifts; for example, $1 \mathrm{~km} \mathrm{~s}^{-1} \leftrightarrow 5 \mathrm{kHz}$ at $1.4 \mathrm{GHz}$, whereas $1 \mathrm{~km} \mathrm{~s}^{-1} \leftrightarrow 1 \mathrm{GHz}$ at $1 \mu \mathrm{m}$. However, dispersion is negligible at optical wavelengths, even at nanosecond timescales (Cordes 2002). Furthermore, natural and cultural sources of nanosecond flashes of significant intensity appear to be entirely absent (Howard \& Horowitz 2001). In other words, a signal that is a spike in the time domain is a natural candidate for interstellar signaling at optical wavelengths, for reasons both scientific and technical. An added bonus is that, at nanosecond timescales, the stellar background becomes negligible.

\subsection{The Case for Optical SETI}

In view of the above, and put most concisely, the primary arguments in favor of conducting SETI at optical (rather than radio) wavelengths are (1) Transmitted beams from optical telescopes are far more slender than their radio counterparts owing to the high gain of optical telescopes. ${ }^{6}$ (2) Dispersion,

\footnotetext{
${ }^{6} 150 \mathrm{~dB}$ for the Keck Telescope at $\lambda=1 \mu \mathrm{m}$ vs. $70 \mathrm{~dB}$ for Arecibo at $\lambda=21 \mathrm{~cm}$, an $80 \mathrm{~dB}$ advantage at optical wavelengths.
}

which broadens radio pulses, is completely negligible at optical frequencies. (3) The capability of radio transmitters has reached a stable maturity, while the power of optical lasers has not yet plateaued and has shown an annual Moore's law doubling, extending over the past 30 years. (4) Natural and cultural backgrounds are negligible (although instrumental backgrounds are significant but manageable in the current optical searches). And finally, (5) the complexity, computational power, and sophistication characteristic of sensitive microwave searches today is unnecessary for optical SETI (OSETI). Detection can be quite simple - a pair of fast, broadband photoncounting detectors in coincidence.

It is also worth noting that scattering and absorption limit the range of transmission in the visible spectrum to a few kiloparsecs (within which there are tens of millions of Sun-like stars); however, at far-infrared wavelengths (as at microwave wavelengths) transmissions can penetrate nearly the entire Galaxy unattenuated. Thus, choice of transmission wavelength may reflect the average separation between civilizations, the number of civilizations in the Galaxy, and, more speculatively, the average lifetime of a civilization (by way of the Drake Equation).

In the following sections we describe the philosophy, design, implementation, and results of such a detector system, in two incarnations: a targeted search, now in its sixth year of continual observations, of some $10^{4}$ Sun-like stars, and a synchronized twin, in its third year of observations, operating at Princeton University.

\section{FEASIBILITY WITH PRESENT TECHNOLOGY}

We do not propose transmission; ${ }^{7}$ nonetheless, it is important to look at the field broadly: if we wanted to transmit, what could we do now, using only "Earth 2000" technology? This is a useful exercise, first to establish plausibility, and then to select an optimum receiving system scenario: among transmit/ receive possibilities, a scheme that works well at both ends is a better bet.

\subsection{Transmitters and Detectors}

Let us consider a civilization, at least as technologically advanced as our own, that wishes to establish contact with its Galactic neighbors. Its task would be to illuminate, with a beacon distinguishable from astrophysical phenomena and from noise, the planetary zones of the nearest $N$ Sun-like stars within some range $R_{\max }$ (comparable to the average separation between intelligent civilizations). In our region of the Galaxy $N \approx 10^{3}$ for $R_{\max }=100 \mathrm{lt}-\mathrm{yr}$ and $N \approx 10^{6}$ for $R_{\max }=1000 \mathrm{lt}-\mathrm{yr}$.

We assume that the transmitting civilization has a catalog of target stars, their positions $\left(\boldsymbol{\theta}_{0}\right)$, proper motions $(\boldsymbol{\mu})$, ranges $(D)$, and radial velocities $\left(v_{r}\right)$ with sufficient accuracy to permit aiming with an error no greater than $\sim 10$ AU when the beam reaches the target. The sky position at which the transmitting civilization must aim $(\boldsymbol{\theta})$ is

$$
\boldsymbol{\theta}=\boldsymbol{\theta}_{0}+\boldsymbol{\mu} \frac{2 D}{c-v_{r}} .
$$

\footnotetext{
7 We justify an asymmetrical listen only strategy by noting that (1) any civilization we could contact is overwhelmingly likely to be more technologically advanced (given evolutionary timescales and the fact that we have only recently crossed the communicative threshold), and (2) because contact with another civilization is unlikely to be its first contact, we would be dealing with a civilization experienced in interstellar communication, perhaps adhering to a Galactic protocol. It therefore seems prudent to devote our limited resources to receiving, not transmitting.
} 
Note that $D /\left(c-v_{r}\right) \approx D / c$ is the light travel time. At $D=$ 1000 lt-yr, 10 AU beaming accuracy corresponds to a proper motion uncertainty of $33 \mu \mathrm{as} \mathrm{yr}^{-1}$ and a positional accuracy of 33 mas. The required range accuracy depends on the star's proper motion; for example, to target the planetary zone (say $10 \mathrm{AU}$ ) of a star whose proper motion is $10 \mathrm{~km} \mathrm{~s}^{-1}$, the range uncertainty cannot exceed 5 lt-yr. These requirements are certainly within the grasp of an advanced civilization, given that our astrometry will achieve microarcsecond precision in the coming decades (Danner \& Unwin 1999), and in any case these accuracies are relaxed if the transmitted beam is broadened to illuminate a larger zone, at the expense of received signal strength.

To send a pulse (or more generally, a packet of information of short duration) to each of $N=10^{6}$ stars with a single-laser system, the sender would probably use an assembly of fast beam-steering mirrors of relatively small size and weight, in combination with a large objective that is steered slowly. Assuming that the sending apparatus could settle to diffraction limited pointing in $\sim 10 \mathrm{~ms}$ (feasible by today's engineering standards), the recipient would observe an optical pulse coming from a nearby star repeated every $10^{4} \mathrm{~s}$. (This period could be dramatically reduced by transmitting only to an intelligently selected subset of the targets and/or by using multiple transmitters; it seems altogether reasonable to expect a pulse period of $10^{3} \mathrm{~s}$ or less.)

The recipient would be able to observe these pulses only if (1) the received fluence per pulse corresponds to at least some tens of photons delivered to the receiving telescope aperture and (2) the flux of laser photons, during the pulse, exceeds the stellar background. It is a remarkable fact, as we show below, that using only Earth 2000 technology we could generate a beamed laser pulse that outshines the Sun by 4 orders of magnitude, in white light, independent of range. One might consider this the "fundamental theorem of optical SETI."

These pulses could be detected with an optical telescope of modest aperture, followed by a beam splitter and a pair of photodetectors of nanosecond or better speed. (We choose nanosecond because it is roughly the speed of photomultiplier tubes, and all known significant backgrounds disappear at this timescale; see Howard \& Horowitz 2001.) The electronics can be as simple as a pair of pulse height discriminators driving a coincidence circuit. The telescope would track the star by the photodetector's "singles" rate while waiting for the unique coincidence signature of some tens of photons arriving in each detector within the resolving time of a nanosecond. As we see below, this signature is easily detected even in broadband visible light; i.e., no spectral filters are required.

\subsection{Backgrounds}

Searches for radio signals of intelligent extraterrestrial origin are plagued by the overwhelming background of radio signals from terrestrial and orbital sources. What is the situation in the optical regime: against what backgrounds must the putative pulsed optical beacon compete?

When detecting light pulses from the neighborhood of a star, the most obvious background is light from the star itself. We circumvent this difficulty by using fast detectors (roughly nanosecond speed), so that the light from the star is just a slow, irregular drumbeat of essentially single photons. A G2 $\mathrm{V}$ star at 1000 lt-yr $\left(m_{V}=12\right)$ delivers $\sim 3 \times 10^{5}$ photons $\mathrm{s}^{-1}$ to a $1 \mathrm{~m}$ telescope. The Poisson-distributed arrivals do not significantly pile up-observing a single photon is rare in a nanosecond and large pileup pulses are greatly suppressed, and by using a beam splitter and a pair of photodetectors in coincidence, the rejection of photon pileup is enhanced further (while additionally suppressing single-detector "hot event" artifacts). ${ }^{8}$ For example, the rate of simultaneous two-photon coincidences in each of a pair of detectors exposed to Poissondistributed arrivals is $R=\left(r_{1}^{2} \tau\right)\left(r_{2}^{2} \tau\right) \tau$, where $r_{1}$ and $r_{2}$ are the individual rates and $\tau$ is the coincidence window. For typical values $r_{1}=r_{2}=10^{5} \mathrm{~s}^{-1}$ and $\tau=1 \mathrm{~ns}$, the coincidence rate caused by simultaneous two-photon pileups in both detectors is $10^{-7} \mathrm{~s}^{-1}$, or less than one pileup per observing year. In other words, light from the parent star itself is unimportant on a nanosecond timescale.

As far as we can tell, both from calculation (Howard \& Horowitz 2001) and from some 16,000 observations conducted so far, there appear to be few astrophysical, atmospheric, or terrestrial mechanisms able to produce events like the nanosecond photon pileup expected from an intentional and powerful pulsed laser beacon. Potential sources we considered include lightning (too extended in time), cosmic-ray induced atmospheric Cerenkov flashes (fast enough, but fluence less than $10^{-3}$ photon per pulse owing to its extended source sky region and large terrestrial footprint), and local effects of the background muon flux (the rate of scintillation events in the optics upstream of the beam splitter plus that of estimated direct ionization events in the detectors themselves produce event rates $\lesssim 10^{-5} \mathrm{~s}^{-1}$ ). We return to these mechanisms in $\S 4.1 .4$ to understand the origin of the few residual events recorded during $\sim 2400 \mathrm{hr}$ at Oak Ridge Observatory.

\subsection{A Transmission Scheme}

To give a sense of the difficulty (or relative ease) of interstellar communication by optical pulses, we calculate several useful quantities for one specific transmission scheme: a "Helios" laser" beamed 1000 lt-yr between two $10 \mathrm{~m} \mathrm{Keck}$ telescopes, each orbiting a Sun-like star. Although this Earth 2000 scheme is surely modest in technological sophistication and scale for a truly advanced civilization, we note that our search reported here is less sensitive than this example, which is given primarily as a plausibility argument.

The transmitted beam is slender as it emerges from the transmitting telescope, $\theta_{b} \approx \lambda_{H} / D_{K}=20$ mas (6 AU at 1000 lt-yr). Its short ( $3 \mathrm{~ns})$ and energetic $\left(E_{p}=4.7 \mathrm{MJ}\right)$ pulses arrive at the receiving telescope, unbroadened in time, as a pulse of

$$
\begin{aligned}
N_{R} & =\frac{\pi^{2} D_{K}^{2} D_{K}^{2} E_{p} 10^{-2 R / 5 R_{E}}}{16 \lambda_{H} R^{2} h c} \\
& =1500 \text { photons. }
\end{aligned}
$$

If the beam is broadened to illuminate a $10 \mathrm{AU}$ disk, then the number of received photons is reduced to $\sim 600$ per pulse. ${ }^{10}$ Here $D_{K}$ is the telescope diameter, $10^{-2 R / 5 R_{E}}=0.87$ is the extinction factor $\left(R_{E} \approx 2 \mathrm{kpc}\right.$ - a rapidly increasing function

\footnotetext{
${ }^{8}$ Dan Werthimer's group at UC Berkeley tested the first pulsed multipledetector system in 1997.

${ }^{9}$ Helios refers to a diode-pumped Yb:S-FAP laser designed at Lawrence Livermore National Laboratory for inertial confinement fusion, potentially capable of generating $3 \mathrm{~ns}, 3.7 \mathrm{MJ}$ pulses $\left(10^{15} \mathrm{~W}\right)$ at $349 \mathrm{~nm}$ (or $4.7 \mathrm{MJ}$ at its native $1.047 \mu \mathrm{m}$ wavelength) at $\sim 10 \mathrm{~Hz}$ rep. rates (Krupke 1997).

${ }^{10}$ For nearby targets, large transmitting apertures, or very short wavelengths, the beam may be too small, given the astrometric errors. Note, however, that the transmitter can adaptively tailor its aperture (and therefore the beam size) so that the photon fluence through the target system is fixed, independent of range.
} 
of wavelength - is the distance over which the intensity of a $1 \mu \mathrm{m}$ pulse will decrease by $1 \mathrm{mag}$ [Mathis 1990]), $\lambda_{H}=$ $1.047 \mu \mathrm{m}$ is the wavelength of the transmitted photons, $R=$ 1000 lt-yr is the distance between the telescopes, and $h$ and $c$ are Planck's constant and the speed of light, respectively. The stellar background is small by comparison, $\sim 3 \times 10^{-2}$ photons $\mathrm{ns}^{-1}$ for a G2 V star (thus $\approx 0.1$ photons during the $3 \mathrm{~ns}$ duration of the laser pulse).

The "pileup" of laser photons here is a desirable situation (unlike normal pulsed observations), because it is the mechanism by which we distinguish an intense laser pulse from Poisson-distributed single photon arrivals from background sources.

The interstellar medium both scatters and absorbs these optical pulses. The effects of scattering over large distances can be quite severe. It tends to reduce the "prompt" pulse height while simultaneously producing two exponential tails, one due to forward scattering (which lasts a few seconds) and a much longer tail due to diffuse scattering (Cordes 2002). The prompt pulse ("ballistic" photons) is unscattered (therefore unbroadened in time) and reduced in amplitude. Absorption acts also to reduce the prompt pulse height, so that the total surviving fraction is $e^{-\tau}$, where $\tau=\left(2 R / 5 R_{E}\right) \ln 10$ is the total optical depth, as mentioned above. Note that the $\sim 13 \%$ extinction (at visible wavelengths) is modest for the range considered above (1000 lt-yr) but becomes unmanageable ${ }^{11}$ for targets within the Galactic disk at distances substantially greater than $R_{E}$.

Thus, in this example the laser outshines its parent star, in broadband visible light, by a factor of $1500 / 0.1$, or approximately $10^{4}$. Moreover, advanced civilizations are expected to be more advanced than we, thus Earth 2000 technology should be a lower bound to the technical sophistication of extraterrestrial civilizations. With a modest extrapolation of another 2-3 orders of magnitude in delivered flux, which can hardly be considered daring given the Moore's law pace of laser technology, we conclude that a moderately advanced civilization should have no trouble outshining its parent star by 6 or more orders of magnitude.

\section{THE TARGETED SEARCH}

Based on the arguments above and their elaborations (which evolved during a set of workshops sponsored by the SETI Institute in 1997-9 [Ekers et al. 2002]), we designed and built a detector system for pulsed laser beacons. It saw first light on 1998 October 19 at Harvard, and has run continually since; a second system began synchronized observations at Princeton on 2001 November $17 .^{12}$

\subsection{How It Works}

The Harvard system rides piggyback on the CfA Digital Speedometer mounted on the $1.5 \mathrm{~m}$ Wyeth Reflector at the Oak Ridge Observatory in the town of Harvard, Massachusetts. The CfA Digital Speedometer supports several dozen research

\footnotetext{
${ }^{11}$ At those ranges, the situation is rescued by the use of infrared wavelengths, $\lambda \approx 2-10 \mu \mathrm{m}$.

${ }_{12}$ Today there are several targeted optical SETI programs, including one at UC Berkeley (Lampton 2000), and a three-detector system at UC Santa Cruz (Wright et al. 2001). Amy Reines and Geoff Marcy examined 577 radial velocity spectra for continuous optical SETI beacons (Reines \& Marcy 2002). Efforts are also underway in Australia (Bhathal 2001) and North Carolina (R. Lodder 2003, private communication).
}

projects, mostly involving radial-velocity measurements of stars (Latham 1992, 2000). Roughly half the light reflected off the entrance slit of the echelle spectrometer (about one third of the total light) is deflected into our photometer, as shown in Figure $1 .{ }^{13}$ Incoming light is reimaged and passes through a beam splitter onto two hybrid avalanche photodiodes ${ }^{14}$ (Hamamatsu R7110U-07), whose outputs feed a pair of multilevel discriminators with levels corresponding to roughly 3 , 6,12 , and 24 photoelectrons. By time stamping level crossings with a LeCroy MTD-135, we obtain approximate "waveforms" of incoming pulses to a precision of $0.6 \mathrm{~ns} .{ }^{15}$ Coincident pulses seen in the two channels trigger the microcontroller to record the arrival time and waveform profiles. Arrival times are recorded twice- by a GPS clock $(0.1 \mu$ s precision and accuracy) and by a computer's internal clock (1 ms precision, but only $\sim 50 \mathrm{~ms}$ accuracy, as determined by comparing many GPS and computer time stamps). A "hot event" veto filters out a class of large-amplitude bipolarity signals that appear to be produced by breakdown events in the photodetectors. Pulse counters, threshold adjusting circuitry, and various controls and monitors allow us to test the apparatus to confirm its stability and proper operation. Fiber-coupled LEDs test the detectors and coincidence electronics before every observation. Figure 2 shows the complete photometer.

Each clear night the CfA Digital Speedometer is used to observe typically $20-50$ stars, with integration times of $2-$ 40 minutes. The observing sequence is determined by the conditions at the telescope and the priorities established in the monthly observing plans. Because of count rate limitations (6000 counts s ${ }^{-1}$ for the Digital Speedometer, typically a factor of 2 higher for the SETI instrument), bright objects are observed only when attenuated (by thin clouds or a neutral density filter) effectively eliminating false events due to photon pileup. Several of the projects involve monitoring variable stars, such as spectroscopic binaries and pulsating stars, so that the targets with the most observations and longest total integration times had been dominated by variable stars and others unlike the Sun. Soon after the Harvard instrument went into operation we established a new observing project designed to provide a large sample of promising SETI targets. The sample of more than 11,000 stars was drawn from the Hipparcos Catalogue and consists of all the main-sequence dwarfs between spectral types late $\mathrm{A}$ and early $\mathrm{M}$ with distances less than $100 \mathrm{pc}$ and declinations between $-20^{\circ}$ and $+60^{\circ}$. Since November, 2001, the Princeton telescope has simultaneously pointed at the same stars on many observing nights. Coordination is achieved by automatically passing target R.A./decl. and other parameters to a Princeton computer, where volunteers point the telescope. During each observation, the diagnostic data, along with coincident pulse data, are sent to a PC and recorded in a log file at each observatory. After each night of observations, the log files are incorporated into a webenabled database to facilitate analysis. We track the data through automated daily e-mails that summarize the previous night's observations. In addition, the web-enabled database allows us

\footnotetext{
13 The Princeton system has full use of its smaller telescope, hence comparable light-gathering aperture; subsequent instrumentation is identical.

${ }^{14}$ HAPDs have the advantage of clean pulse height discrimination, at the price of increased corona discharge, as compared with traditional lower voltage multistage photomultiplier tubes.

${ }^{15}$ As configured, the LeCroy chip only timestamps the last upward and downward crossing for each level, thus the waveforms of more complicated shapes (e.g., double pulses) cannot be completely reconstructed.
} 


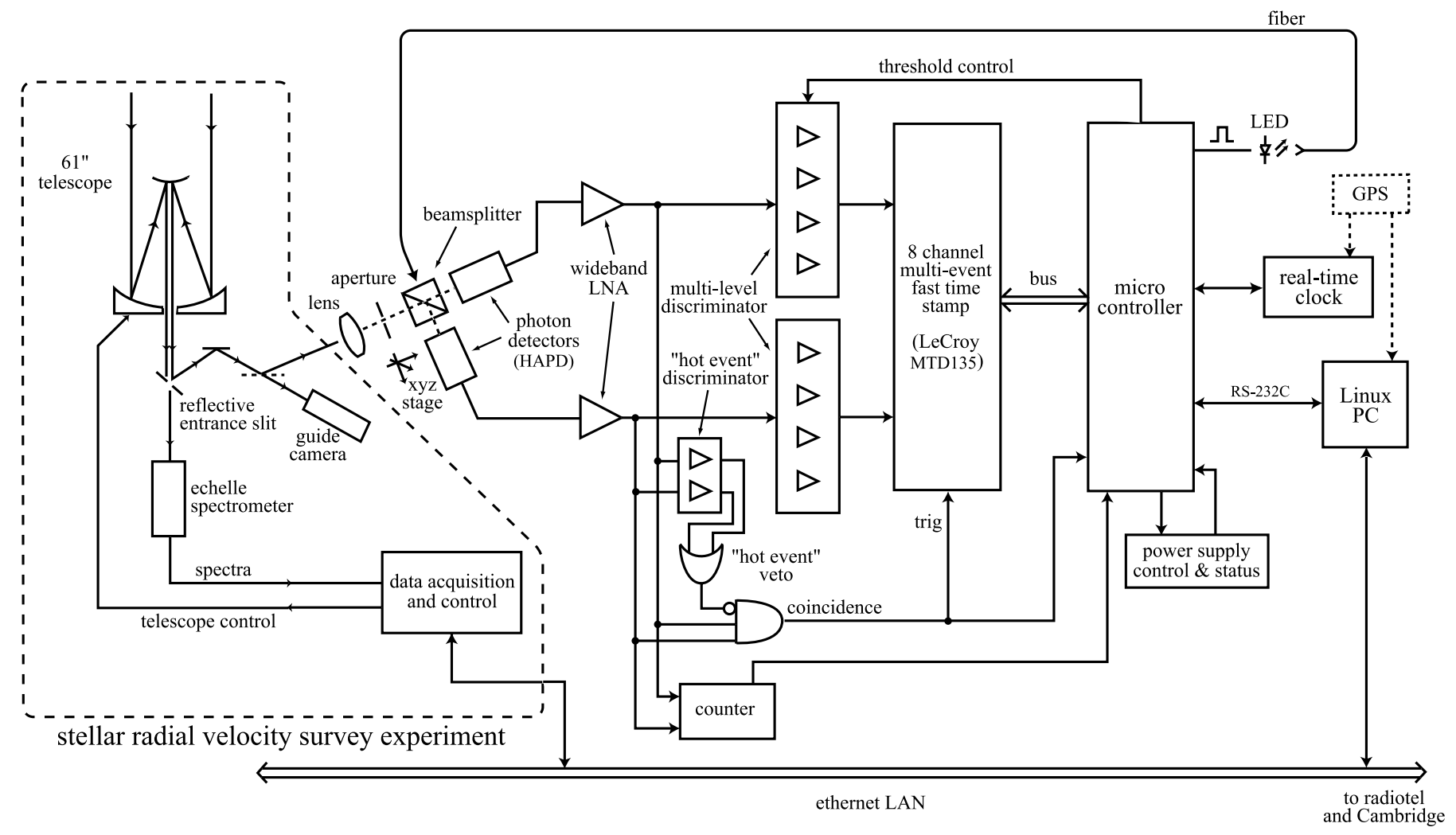

Fig. 1.-Block diagram of the Harvard targeted optical pulse detector. Unused light from the echelle spectrograph is imaged onto a pair of hybrid avalanche photodetectors, whose coincidence triggers fast time-stamping of waveform crossings through four preset levels. Complete circuit schematics are available (Horowitz 2004).

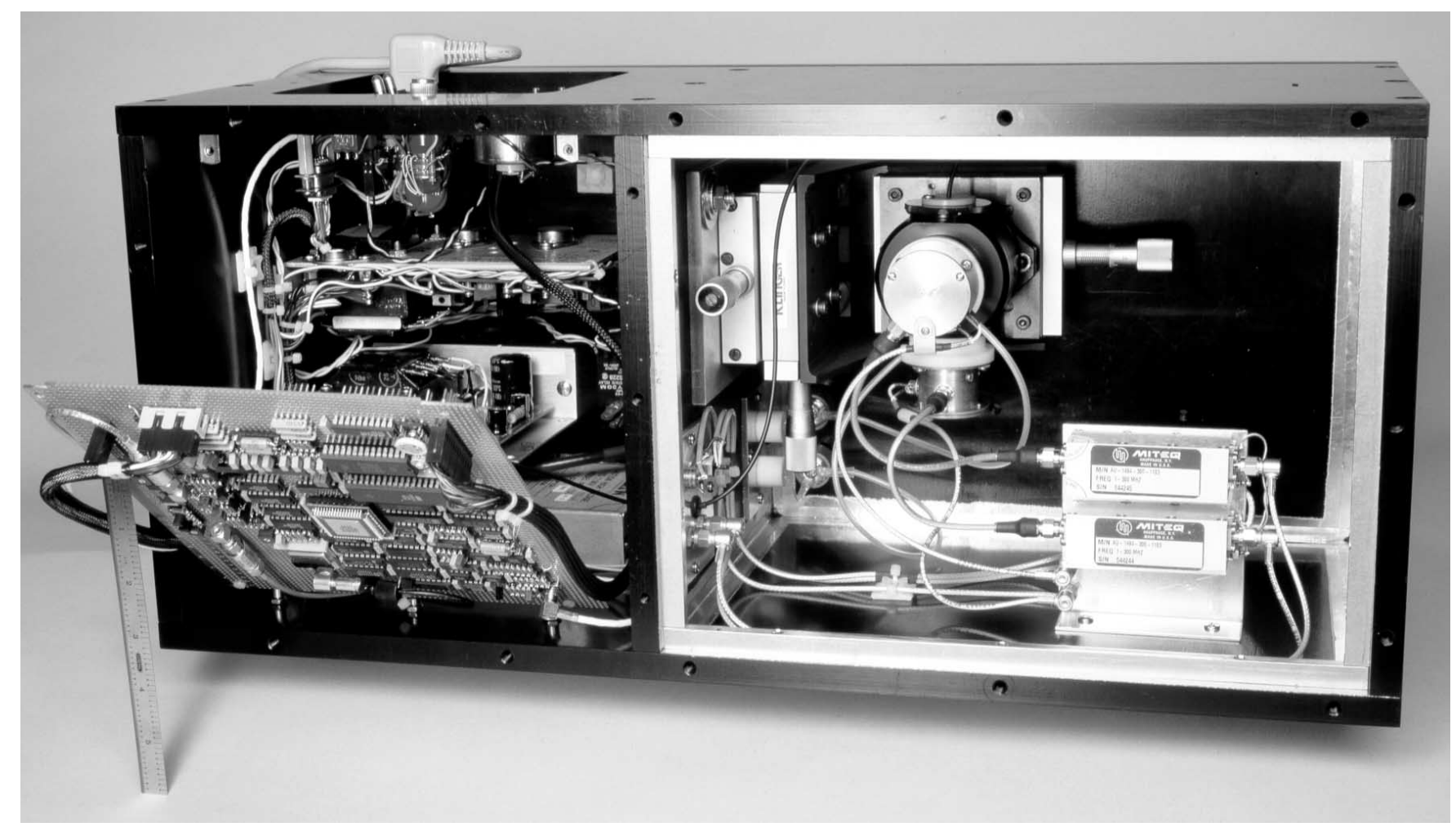

FIG. 2.-Harvard targeted search photometer, with covers removed. Light enters from the rear of the righthand compartment, focused onto a 30 arcsec aperture, then passes through a beam splitter onto the pair of HAPDs on their 3-axis stage. The detectors run at a gain of $\sim 4 \times 10^{4}$, producing $\sim 50 \mu \mathrm{V}$ negative pulses into $50 \Omega$, which are amplified and sent to the electronics in the lefthand compartment. The latter perform coincidence, 4-level ADC, timing, logging, hot-event veto, and communication with the host Linux PC. The photometer measures $25 \times 25 \times 60 \mathrm{~cm}$, and weighs $30 \mathrm{~kg}$. 
easily to view the data in many forms: chronological summaries, ordered searches by various criteria (e.g., total events, event rate, total observation time), observational summaries for individual objects, diagnostic data for particular observations, etc. Further details are available at Coldwell (2002) and Horowitz (2004).

\subsection{Sensitivity}

We estimate the sensitivity of the Harvard instrument by following a light pulse through the entire system (Fig. 1). The optical path includes four reflections (each $\sim 85 \%$ efficient), a lens $(\sim 92 \%)$, and a beam splitter $(\sim 92 \%)$. One of the mirrors is the entrance slit to the echelle spectrometer, which reflects roughly one-third of the light into the OSETI instrument on average, depending on seeing. The beam splitter sends half the light to each hybrid avalanche photodiode (HAPD), which has a broad $\sim 20 \%$ plateau in quantum efficiency (QE) for $\lambda=450-650 \mathrm{~nm}$ (and QE $>1 \%$ for $\lambda=350-720 \mathrm{~nm}$ ). The signal must exceed the lowest threshold (three photoelectrons) in each detector during the discriminator's averaging time (5 ns). Accounting for these factors, the Harvard instrument's threshold sensitivity is 100 optical photons $(\lambda=$ $450-650 \mathrm{~nm}$ ) per square meter, arriving at the telescope in a group within $5 \mathrm{~ns}$.

The Princeton instrument is identical, except that it receives all of the light from its $0.9 \mathrm{~m}$ primary mirror, and the light path includes only three mirrors. Multiplying the appropriate factors, the Princeton instrument is sensitive to signals of 80 optical photons $(\lambda=450-650 \mathrm{~nm})$ per square meter arriving in a group within $5 \mathrm{~ns}$. With both instruments simultaneously observing, Princeton can veto a Harvard signal, although the confidence in this veto depends on the signal intensity at Harvard and the observing conditions at the two sites.

In summary, the project parameters of targeted OSETI at Harvard and Princeton are as follows.

Telescopes.-(a) $1.6 \mathrm{~m}$ Wyeth telescope in Harvard, Massachusetts-1/3 of light used for OSETI, (b) 0.9 m FitzRandolph telescope in Princeton, New Jersey-all light used for OSETI.

Photometers.-(a) Beam-split pair of hybrid avalanche photodiodes (350-720 nm response, peaked between 450 and $650 \mathrm{~nm}$ ), (b) Pulse amplitude profiles recorded to $0.6 \mathrm{~ns}$ resolution, (c) GPS-derived time stamping of events to $0.1 \mu \mathrm{s}$ at each observatory.

Objects observed.-(a) 15,897 observations of 6176 stellar objects with Harvard instrument, (b) 1721 simultaneous observations of 1142 stellar objects with Princeton and Harvard instruments, (c) Objects selected for radial velocity surveysmany FGK dwarfs.

Sensitivity. -100 photons $\mathrm{m}^{-2}$ in the photometers' waveband and aperture in $\lesssim 5 \mathrm{~ns}$ ( 80 photons $\mathrm{m}^{-2}$ for Princeton).

\section{RESULTS OF FIVE YEARS' OBSERVATIONS}

\subsection{Harvard Observations}

From 1998 October through 2003 November, the targeted search with the Harvard instrument performed 15,897 observations of 6176 stars, for a total of $2378 \mathrm{hr}$ of observation. Our target list is composed of objects being surveyed both for SETI and for other astrophysical interests. Two of the authors (D. L. and R. S.) are characterizing $\sim 11,000 \mathrm{~F}, \mathrm{G}$, and $\mathrm{K}$ dwarfs (2079 observed at least once with the OSETI instrument) for possible observations by next-generation targeted

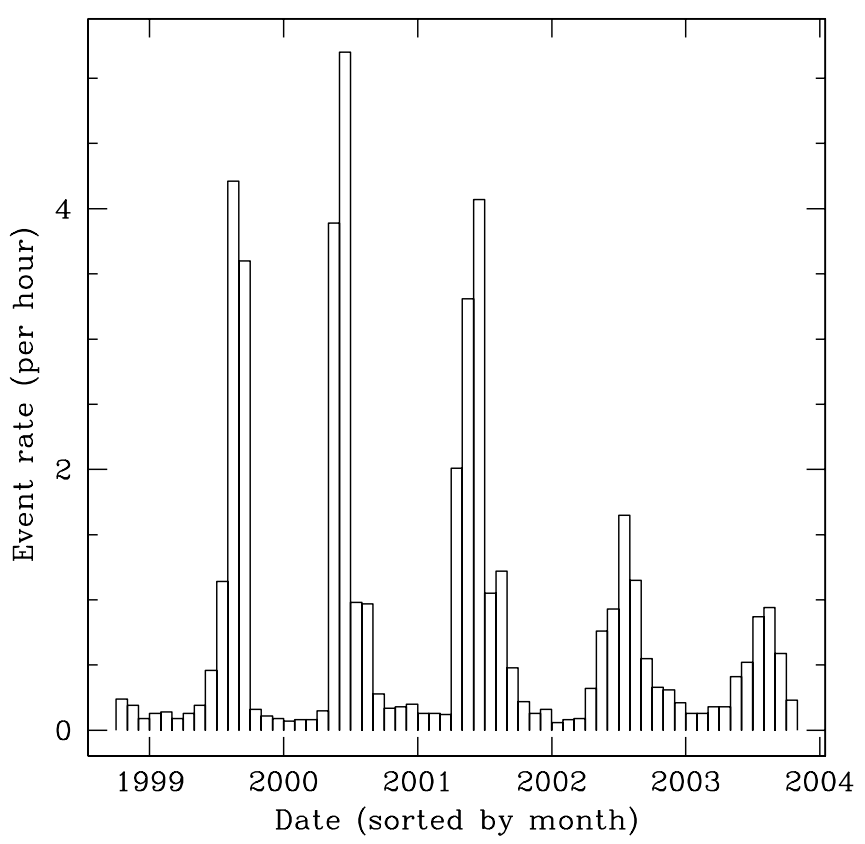

FIG. 3.-Seasonal variation in the event rate at Harvard. Corona breakdown substantially elevates the rate during the humid summer months. This plot includes all observations, regardless of quality.

microwave SETI, seeking evidence of stellar companions that would prohibit planets in the habitable zone. The radial velocities of a sample of $\sim 3000$ nearby $G$ dwarfs are being monitored to characterize the population of stellar companions with spectroscopic orbits. Other programs observe a variety of additional targets (very young stars, A dwarfs, and very old stars in the solar neighborhood, among others).

In the five years of observations, we recorded 4746 " triggers" (i.e., instances when the lowest thresholds are simultaneously exceeded in both channels). Although all triggers are recorded, the reported waveforms are passed through a filter that enforces certain validity checks: the signals seen in each channel must be roughly the same amplitude (within one level of each other), and they must overlap in time. The subset of triggers that pass this test are labeled "events"; to date, we have registered 1117 events. This filter is unlikely to exclude a genuine pulsed flash - the LED test flashes, which are done before every observation, pass this test with only rare exceptions.

Since the 1117 events are distributed impartially among 6176 objects $(\S 4.1 .3)$, we have confidence that the majority of the events arise from natural causes. Furthermore, in attempts to identify their source, we logged events even during tests with the observatory dome closed. Clearly, instrumental effects contribute background events. In the analysis that follows, we attempt to remove the instrumental backgrounds from the Harvard data to look for residual events, possibly of extraterrestrial origin. We also examined the Harvard observations during which Princeton provided verification through simultaneous observations; with one possible exception, we found no events synchronously occurring at the two observatories $(\S 4.3)$.

\subsubsection{Seasonal Variation}

A histogram of the event rate by month (Fig. 3) reveals the largest source of background events, evidenced by a marked systematic seasonal trend in the event rate, apparently due to 
ambient humidity. During the dry months of fall, winter, and early spring (October-April), the data exhibit an event rate of about $0.16 \mathrm{hr}^{-1}$ and a trigger rate of about $0.5 \mathrm{hr}^{-1}$. However, the event rates are 30-40 times higher during the warmer and more humid summer months (May-September), as shown. Furthermore, we see a memory effect: observations following wet weather exhibit event rates many times higher than the summer average but recover after 1-2 nights of dry weather. Opening the camera (which is normally kept tightly closed and flushed with dry nitrogen) for maintenance work similarly raises event rates, but with a longer recovery time ( $\sim 15$ days). These events tend to cluster in time with, say, 10 events in 3 minutes followed by many tens of minutes of quiet. These symptoms all point to corona discharge, a high-voltage breakdown characterized by radio-frequency and optical emission. We mitigated the humidity effect beginning in 2002 by adding dry nitrogen gas lines, bakeout heaters, and an entrance window, but these efforts reduced the summer event rate only by a factor of 2 or 3 (see Fig. 3).

We examined, and excluded, the possibility that the seasonal variation was due to temperature-dependent gain in the HAPDs: the HAPD bias power supply (Power Technology PD-3) includes temperature compensation matched to the detector characteristics, and furthermore, the observed pulse waveforms produced by the HAPDs during hot and cold weather are indistinguishable. In addition, the event rate shows no statistically significant correlation with temperature.

\subsubsection{Data Sets}

Throughout this paper, we refer to the three data sets shown in Table 1: $\mathrm{DS}_{\text {all }}$ is the data set used above and consists of all observations made from Harvard. $\mathrm{DS}_{\text {clean }}$ is a subset of $\mathrm{DS}_{\text {all }}$ from which nights with anomalous trigger rates have been removed. To be excluded from $\mathrm{DS}_{\text {all }}$, a night's observations must have a trigger rate greater than one per hour, and two or more events spread among two or more objects. Although this cut may seem arbitrary, in practice it cleanly removes nights with corona-polluted data. The data excluded from this set were scrutinized for clear extraterrestrial beacons (e.g., a pulse train of events for one object). $\mathrm{DS}_{\text {overlap }}$ is the subset of $\mathrm{DS}_{\text {all }}$ during which Princeton jointly observed (see $\S 4.3$ ). Roughly $60 \%$ of the objects in these data sets are Sun-like stars (late F through early $\mathrm{M}$ ).

\subsubsection{Consistent with Poisson Statistics}

$\mathrm{DS}_{\text {clean }}$ includes 11,600 observations, summarized in Tables 1 and 2. Of the 4730 objects observed, 95\% (4496) had no events at all. Note particularly that among the objects with events, those with more events were observed for longer durations (Tables 2 and 3) and more often (Table 4). The 274 events were distributed among 234 separate objects as shown in the "Observed" column of Table 2. The objects at the tail of this distribution are particularly interesting-20 objects with two events, four objects with three events, and four objects with four events. Do any of the objects show an extrastatistical number of events? Is there any evidence of periodicity? Is this distribution consistent with any model?

The events in $\mathrm{DS}_{\text {clean }}$ were modeled with a Monte Carlo simulation of the observations using Poisson statistics. We assumed that the event rate was constant $\left(0.16 \mathrm{hr}^{-1}\right.$, to generate the observed total of 274 events in $1721 \mathrm{hr}$ ) - as one would expect for a random physical process (e.g., radioactivity, cosmic rays) unrelated to the telescope's target - and calculated the average number of objects with $0,1,2, \ldots$,
TABLE 1

Summary of Data from Harvard Search

\begin{tabular}{|c|c|c|c|}
\hline Parameter & $\mathrm{DS}_{\mathrm{all}}$ & $\mathrm{DS}_{\text {clean }}$ & $\mathrm{DS}_{\text {overlap }}$ \\
\hline 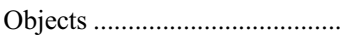 & 6176 & 4730 & 1142 \\
\hline 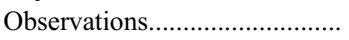 & 15,897 & 11,600 & 1721 \\
\hline Observations per object ........... & 2.1 & 2.5 & 1.5 \\
\hline Integration $(\mathrm{hr}) \ldots \ldots \ldots \ldots \ldots \ldots \ldots$ & 2378 & 1721 & 244 \\
\hline Integration per object $(\mathrm{hr}) \ldots \ldots$ & 0.39 & 0.37 & 0.21 \\
\hline 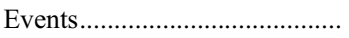 & 1117 & 274 & 130 \\
\hline Event Rate $\left(\mathrm{hr}^{-1}\right) \ldots \ldots \ldots \ldots \ldots \ldots$ & 0.47 & 0.16 & 0.53 \\
\hline 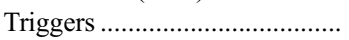 & 4746 & 1066 & 614 \\
\hline Trigger Rate $\left(\mathrm{hr}^{-1}\right) \ldots \ldots \ldots \ldots \ldots$ & 2.00 & 0.62 & 2.52 \\
\hline
\end{tabular}

NoTES.-Summary statistics from the Harvard search for three data sets: $\mathrm{DS}_{\text {all }}$ consists of all observations made from Harvard. $\mathrm{DS}_{\text {clean }}$ is a subset of $\mathrm{DS}_{\text {all }}$ with certain high trigger rate nights removed (see $\S 4.1 .2$ ). $\mathrm{DS}_{\text {overlap }}$ is the subset of $\mathrm{DS}_{\text {all }}$ during which Princeton jointly observed (see $\S 4.3$ ). Princeton contributed 429 events and 2327 triggers to $\mathrm{DS}_{\text {overlap. }}$

events during 10,000 runs (with standard deviations in the averages giving the error bars), as shown in "Simulated Poisson" in Table 2. The Monte Carlo event distribution was verified by direct calculation of the Poisson probabilities for each object having $0,1,2, \ldots$, events and summing over objects. Since the total integration time per object varied substantially (Table 3), the calculation and Monte Carlo simulation used the actual distribution of observing times.

The observed event distribution in Table 2 appears consistent with the model of uniform background rate. The slight discrepancy between the observed and modeled event distributions - more observed objects with four events - can be plausibly explained by postulating that the observations during a few corona-plagued nights (with their highly variable event rates) were included in $\mathrm{DS}_{\text {clean }}$. The objects with multiple events are nevertheless of interest and are discussed further in $\S 4.2$.

\subsubsection{Other Sources of Events?}

What, then, caused these 274 Poisson distributed events? Low-level corona is a plausible explanation, but several others warrant investigation as well.

Cosmic-ray muons (and other charged particles) are a potential source of events. Could a muon traveling by chance down the axis of the telescope produce enough beamed Cerenkov radiation to be detected by the OSETI instrument? Cosmic-ray muons have an average energy of $2 \mathrm{GeV}$ and a flux of $I(\phi)=I_{\nu} \cos ^{2} \phi$, where $\phi$ is the zenith angle and $I_{\nu}=$ $8 \times 10^{-3} \mathrm{~cm}^{-2} \mathrm{~s}^{-2} \mathrm{sr}^{-2}$ (Rossi 1948). The number of photons from a single muon is a function of its energy (with a threshold energy of $4.3 \mathrm{GeV}$ ), and its path length is described by $d^{2} N / d E d x=370 \sin ^{2} \theta_{C}(E) \mathrm{eV}^{-1} \mathrm{~cm}^{-1}$, where $\theta_{C}=$ $(\beta n)^{-1}, \beta=v_{\mu} / c$, and $n$ is the index of refraction. At sea level, this is $\sim 10$ optical $(2 \mathrm{eV})$ photons $\mathrm{m}^{-1}$. Although the light cone's opening angle is small $\left[\theta_{C}(E)<1.4\right.$ for air], most of the photons either miss the telescope or do not couple into the instrument, even for muons traveling down the telescope axis.

Figure 4 shows the probability density of events and observations as a function of telescope zenith angle for a restricted set of $\mathrm{DS}_{\text {clean }}$ with a particularly low event rate $(9400$ observations of 3928 objects with an event rate of $0.15 \mathrm{hr}^{-1}$ ). If Cerenkov photons were a significant background, we would expect to see excess probability for events at low zenith angles, which we do not. A histogram derived from triggers 
TABLE 2

Harvard Observations: Distribution of Events

\begin{tabular}{|c|c|c|c|c|c|c|c|}
\hline \multirow[b]{2}{*}{ EVEnTs } & \multicolumn{2}{|c|}{ ОвлестS } & \multicolumn{2}{|c|}{ OBSERVATIONS } & \multicolumn{2}{|c|}{ InTEGRATION TIME (hr) } & \multirow[b]{2}{*}{ EVENTS PER Hour } \\
\hline & Observed & Simulated Poisson & Total & Per Object & Total & Per Object & \\
\hline 0 & 4496 & $4495 \pm 14$ & 9518 & 2.1 & 1327 & 0.3 & 0 \\
\hline 1. & 206 & $207 \pm 14$ & 1378 & 6.7 & 257 & 1.3 & 0.80 \\
\hline 2 & 20 & $21 \pm 4.4$ & 418 & 21 & 75 & 3.8 & 0.53 \\
\hline 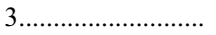 & 4 & $4.9 \pm 2.0$ & 81 & 20 & 14 & 3.5 & 0.86 \\
\hline 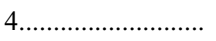 & 4 & $1.5 \pm 1.1$ & 205 & 51 & 48 & 12 & 0.33 \\
\hline $5+\ldots \ldots \ldots \ldots \ldots \ldots \ldots \ldots$ & 0 & $0.7 \pm 0.8$ & 0 & 0 & 0 & 0 & 0 \\
\hline Total (274) ...... & 4730 & 4730 & 11600 & 2.5 & 1721 & 0.37 & 0.16 \\
\hline
\end{tabular}

Notes.-DS clean $_{\text {s. }}$ sorted by events per object. Note that $95 \%$ of the objects (4496/4730) do not have any events. The expected number of objects - derived from a calculation based entirely on the event rate and the distribution of integration times-correlates well with the actual number of events. Also note that both the number of observations and integration time per object is much higher for multiple-event objects, as one would expect for randomly distributed events.

(instead of events) from the same data set (not shown) is qualitatively similar. Thus, we conclude from both calculation and observation that Cerenkov flashes do not contribute events.

Another potential source of the residual events is energetic muons traveling through both HAPDs. Such muons would have energy sufficient to eject electrons from the photocathodes, which would be amplified and detected. However, it would require a lucky hit for a single muon to pass through both photodetectors: estimating the relevant detector cross sections to be $0.25 \mathrm{~cm}^{2}$, a simple geometric calculation shows that muons traverse both detectors at a rate of $\sim 10^{-5} \mathrm{~s}^{-1}$, or once every $\sim 25 \mathrm{hr}$. We calculated the "detector zenith angle" (the angle between the vector connecting the two detectors and the zenith) for each observation to test for the excess events that would be expected at small zenith angles, owing to the $\cos ^{2} \phi$ dependence of muon flux. Figure 5 shows the probabilities of events and observations as a function of the detector zenith angle for the same data set used for Figure 4. We see no evidence of excess events due to this mechanism. Using triggers instead of events in Figure 5 leads to a qualitatively similar histogram.

Cerenkov radiation from muons passing through the $25 \mathrm{~mm}$-cube glass beam splitter is another potential source of events. For glass $\left(n=1.5, \theta_{C}=0.84 \mathrm{rad}\right), \sim 500$ visible-

TABLE 3

Distribution of Integration Times

\begin{tabular}{|c|c|c|c|c|c|}
\hline \multirow{2}{*}{$\begin{array}{l}\text { INTEGRATION } \\
\text { (minutes) }\end{array}$} & \multirow[b]{2}{*}{ Овлестs } & \multicolumn{4}{|c|}{ EVENTS } \\
\hline & & 0 & 1 & 2 & $3+$ \\
\hline $0-1$ & 36 & 36 & 0 & 0 & 0 \\
\hline $2-3 \ldots \ldots \ldots \ldots \ldots \ldots \ldots-1 . \cdots \cdots$ & 1207 & 1203 & 4 & 0 & 0 \\
\hline $4-7 \ldots \ldots \ldots \ldots \ldots \ldots . .$. & 1048 & 1027 & 21 & 0 & 0 \\
\hline $8-15 \ldots \ldots \ldots \ldots \ldots \ldots \ldots \ldots . .$. & 876 & 845 & 29 & 2 & 0 \\
\hline $16-31$ & 821 & 783 & 36 & 1 & 1 \\
\hline 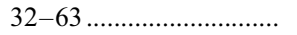 & 410 & 355 & 53 & 1 & 1 \\
\hline 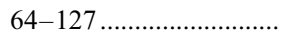 & 211 & 172 & 33 & 6 & 0 \\
\hline $128-255 \ldots \ldots \ldots \ldots \ldots \ldots \ldots$ & 83 & 61 & 19 & 3 & 0 \\
\hline 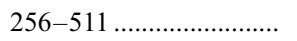 & 27 & 14 & 6 & 4 & 3 \\
\hline $512+\ldots \ldots \ldots \ldots \ldots \ldots \ldots \ldots \ldots$ & 11 & 0 & 5 & 3 & 3 \\
\hline
\end{tabular}

Notes.-Distribution of integration times in $\mathrm{DS}_{\text {clean }}$. Note that on average the objects with multiple events were observed for longer than those objects with zero or one event. wavelength photons are produced per muon per $\mathrm{cm}$ traveled. It would require a lucky hit to deliver photons from the beam splitter to both detectors, but it seems plausible that this scenario could produce events. Scintillation in the beam splitter glass from muons or radioactive decay products is unlikely to trigger an event due to the small size of the beam splitter and the low scintillation yield of glass. Muon capture in the beam splitter (where it would energetically decay) is unlikely given the low capture cross section and small beam splitter size. To test these beam splitter scenarios, however, we temporarily replaced the cubical beam splitter with a thin $(1 \mathrm{~mm})$ coated plate beam splitter. During several nights of tests, we observed no statistically significant change in event rate.

The Cerenkov radiation and scintillation scenarios described above, as well as other potential backgrounds, are discussed in Howard \& Horowitz (2001).

\subsection{Interesting Objects and Reobservations}

Tables 5 and 6 show the objects that have two or more events in $\mathrm{DS}_{\text {clean }}$, their observing and event/trigger statistics, and astronomical information. We examined the observational histories of these objects for indications that the events from one or more of them was due to intentional extraterrestrial communication - an extrastatistical number of events, a clustering of an object's events in one night's observations, or simultaneous event detection at Harvard and Princeton. Objects with any of these characteristics were concurrently reobserved by Harvard and Princeton ("Reobservations" in Table 5). These reobservations are not included in the three data sets in Table 1.

TABLE 4

Distribution of OBservations

\begin{tabular}{|c|c|c|}
\hline Observations & Objects & Events \\
\hline 1 & 3223 & 65 \\
\hline 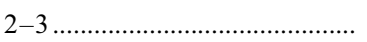 & 767 & 51 \\
\hline $4-7 \ldots \ldots \ldots \ldots$ & 437 & 55 \\
\hline 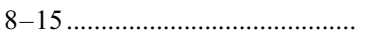 & 239 & 46 \\
\hline $16-31$ & 42 & 19 \\
\hline 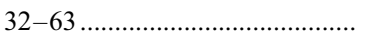 & 19 & 35 \\
\hline 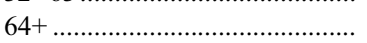 & 3 & 3 \\
\hline
\end{tabular}

NoTE.-Summary of the distributions of events and objects from observations in $\mathrm{DS}_{\text {clean }}$. 


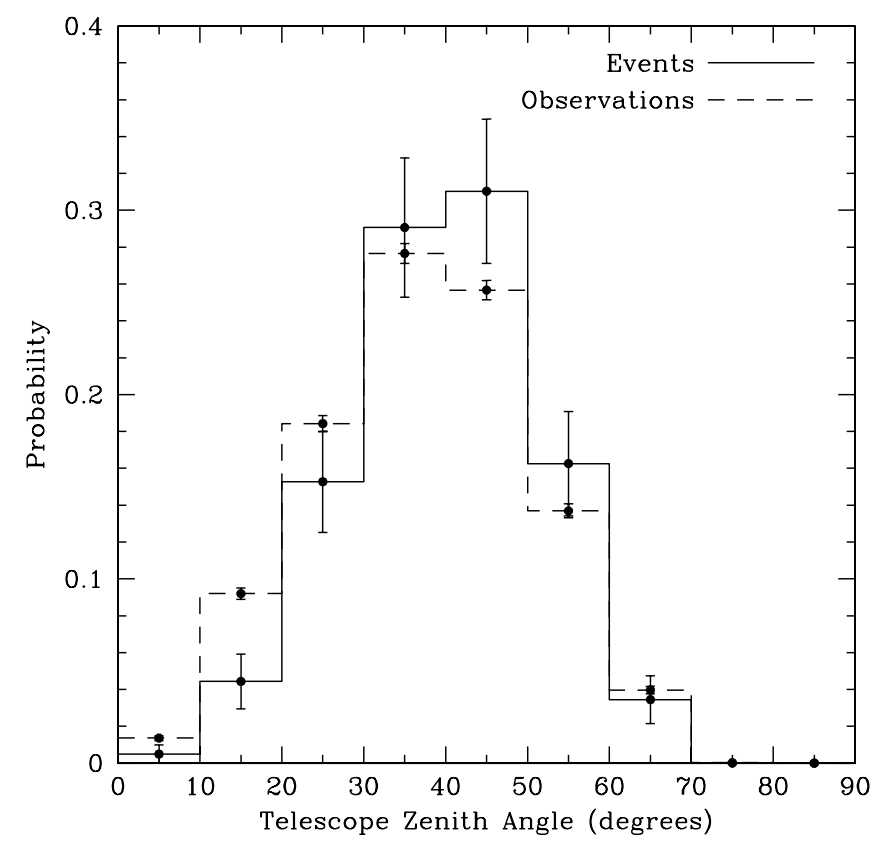

FIG. 4. - Histogram of the probability of events (solid) and observations (dashed) at Harvard as a function of the telescope zenith angle for a restricted set of $\mathrm{DS}_{\text {clean }}$. If a significant fraction of the events in this data set were due to Cerenkov radiation from muons traveling down the line of sight of the telescope, then the probability of events would be concentrated at small telescope zenith angles.

Note that many of the objects in Tables 5 and 6 (particularly those with three and four events) were observed often and have long total observation times, typically because they are suspected short-period binaries or are variable on short timescales. These objects are therefore deemed less likely hosts for intelligent civilizations. Although many Sun-like stars were observed $(\sim 60 \%$ of the objects and observation time in $\mathrm{DS}_{\text {clean }}$ ), few are represented in Tables 5 and 6, because they had less observing time per object; that is, Tables 5 and 6 select for objects with long total observing times, which tend not to be Sun-like. Nevertheless, given the consequences of a confirmed nanosecond pulse, a careful analysis is warranted.

\subsubsection{HD 220077}

The three triggers (all events) recorded by HD 220077 on 2000 November 4 warrant the greatest attention. ${ }^{16}$ The three events were recorded during 10 minutes spread over five observations. The experiment ran for only 46 minutes that night, and none of the 10 other objects registered an event (although two triggered), as shown in Figure 6. Although this night was the first time the experiment had run in 25 days, and it did not run during the five nights following, all diagnostic data (count rates, temperature, weather, etc.) appear normal. The event rates during autumn of 2000 were relatively low $\left(\sim 0.15-0.20 \mathrm{hr}^{-1}\right)$.

The time differences between successive events $\left(\tau_{1}=914 \mathrm{~s}\right.$ and $\tau_{2}=289 \mathrm{~s}$ ) do not appear to be part of a (perhaps

\footnotetext{
${ }^{16}$ We use Universal Time (UT), and star names from the CfA Digital Speedometer surveys, which in some cases are not in common use. Other names for objects (such as Henry Draper or Hipparcos numbers) and celestial coordinates are given in Table 6.
}

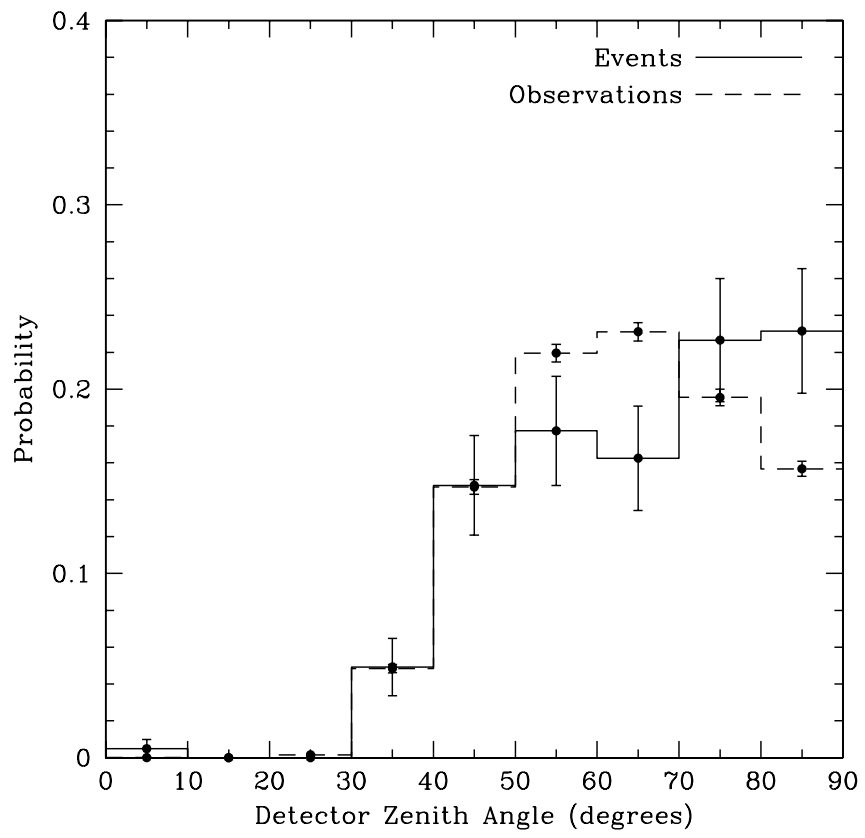

FIG. 5.-Histogram of the probability density of events (solid) and observations (dashed) at Harvard as a function of the "detector zenith angle" - the angle between the vector connecting the two detectors and the zenith-for a restricted set of $\mathrm{DS}_{\text {clean. }}$. If a significant fraction of the events in this data set were due to muons passing through both detectors, then the probability of events would be concentrated at small detector zenith angles.

incompletely sampled) regular pattern: $\tau_{1} / \tau_{2} \neq n / m$ for small integers $n$ and $m$ (which we call the "Rational Period Test"), even when allowing for the \pm 1 s clock accuracy in 1999 (before the GPS clock was added).

As shown in Table 7, HD 220077 was observed 15 times over 50 minutes in $\mathrm{DS}_{\text {all }}$. One additional event was recorded on 2003 July 15 (in $\mathrm{DS}_{\text {all }}$, but not $\mathrm{DS}_{\text {clean }}$ ), however the trigger and event rates were both elevated that night. This object was jointly reobserved by Harvard and Princeton for 99 minutes and no events or triggers were seen at Harvard.

In general, one can calculate the Poisson probability of recording no events in reobservations of a given duration, assuming that an event rate from previous observations should apply. We calculate two such "reobservation probabilities," $p_{r_{1}}$ and $p_{r_{2}}$, using the event rates for HD 220077 from 2000 November $4\left(r_{1}=0.33\right.$ minute $\left.^{-1}\right)$, and for all observations of HD 220077 in $\mathrm{DS}_{\text {clean }}\left(r_{2}=0.14\right.$ minute $\left.^{-1}\right)$, respectively, and the reobservation times listed in Table 5. Although instructive, $p_{r_{1}} \sim 10^{-14}$ and $p_{r_{2}} \sim 10^{-6}$ are probably unrealistically low since an event rate is poorly defined by so few events - a constant rate may not even be an accurate characterization of a process with such limited statistics. Nevertheless, it remains unlikely that a natural or artificial source would produce three events in 10 minutes, and then no events for nearly 10 times as long. We conclude that the events in question were probably a statistical fluctuation of background processes. The strength of this conclusion is limited, however, given the modest time spent reobserving. Additional reobservations (for, say, tens of hours) could test this conclusion.

HD 220077 is an F7 V dwarf with a visual companion $0.25 \mathrm{mag}$ fainter at a separation of 0.23 . The $38 \mathrm{CfA}$ radial velocities for the composite light of both stars show a hint of a slow drift over the observed span of 4147 days, which supports 
TABLE 5

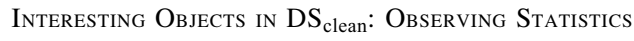

\begin{tabular}{|c|c|c|c|c|c|c|c|c|c|c|c|}
\hline \multirow[b]{2}{*}{ ОвЈест } & \multicolumn{7}{|c|}{ OBSERVATIONS IN DS $\mathrm{D}_{\text {clean }}$} & \multicolumn{4}{|c|}{ REOBSERVATIONS } \\
\hline & Events & Triggers & Observations & Hours & Rate & Multiple Events/Triggers? & Veto? $(\mathrm{E} / \mathrm{T})$ & Hours & Events & Triggers & Observations \\
\hline HD $14535 \ldots \ldots \ldots \ldots \ldots \ldots$ & 4 & 7 & 52 & 13.3 & 0.30 & $\ldots$ & $0 / 1$ & $\ldots$ & $\ldots$ & $\ldots$ & $\ldots$ \\
\hline$\phi$ CYG $\ldots \ldots \ldots \ldots \ldots \ldots \ldots \ldots \ldots \ldots \ldots \ldots \ldots$ & 4 & 10 & 59 & 7.6 & 0.53 & $\ldots$ & $\ldots$ & $\ldots$ & $\ldots$ & $\ldots$ & $\ldots$ \\
\hline 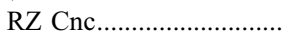 & 4 & 7 & 54 & 15.6 & 0.26 & $\ldots$ & $0 / 0$ & $\ldots$ & $\ldots$ & $\ldots$ & $\ldots$ \\
\hline 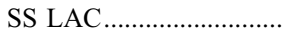 & 4 & 8 & 40 & 11.7 & 0.34 & $\ldots$ & $\ldots$ & $\ldots$ & $\ldots$ & $\ldots$ & $\ldots$ \\
\hline 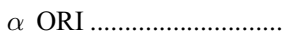 & 3 & 16 & 40 & 4.4 & 0.68 & $2 / 3$ on 2002 Nov 8 & $\ldots$ & 1.0 & 1 & 4 & 3 \\
\hline 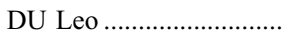 & 3 & 8 & 27 & 8.3 & 0.36 & 3/7 on 1999 May 31 & $\ldots$ & 2.2 & 0 & 0 & 7 \\
\hline 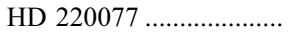 & 3 & 3 & 9 & 0.3 & 8.82 & $3 / 3$ on 2000 Nov 4 & $\ldots$ & 1.7 & 0 & 0 & 6 \\
\hline LSR2-1471 ..................... & 3 & 3 & 5 & 1.0 & 3.11 & $\ldots$ & $\ldots$ & $\ldots$ & $\ldots$ & $\ldots$ & $\ldots$ \\
\hline $\mathrm{BD}+18^{\circ} 2930 \ldots \ldots \ldots \ldots \ldots$ & 2 & 2 & 1 & 0.3 & 6.00 & $2 / 2$ on 1999 Feb 15 & $\ldots$ & 1.7 & 0 & 0 & 5 \\
\hline 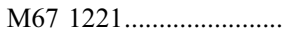 & 2 & 2 & 8 & 2.0 & 1.01 & $\ldots$ & $\ldots$ & $\ldots$ & $\ldots$ & $\ldots$ & $\ldots$ \\
\hline 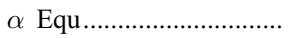 & 2 & 4 & 14 & 1.2 & 1.70 & $\ldots$ & $0 / 2$ & $\ldots$ & $\ldots$ & $\ldots$ & $\ldots$ \\
\hline $\mathrm{BD}+61^{\circ} 1045 \ldots \ldots \ldots \ldots \ldots$ & 2 & 2 & 6 & 1.6 & 1.23 & $\ldots$ & $\cdots$ & $\ldots$ & $\ldots$ & $\ldots$ & $\ldots$ \\
\hline Capella ............................... & 2 & 8 & 37 & 5.4 & 0.37 & $\ldots$ & $\cdots$ & $\ldots$ & $\ldots$ & $\ldots$ & $\ldots$ \\
\hline EU Del ............................ & 2 & 9 & 72 & 7.1 & 0.28 & $\ldots$ & $1 / 2$ & $\ldots$ & $\ldots$ & $\ldots$ & $\ldots$ \\
\hline G65-43 „......................... & 2 & 2 & 7 & 1.9 & 1.05 & $\ldots$ & $\ldots$ & $\ldots$ & $\ldots$ & $\ldots$ & $\ldots$ \\
\hline 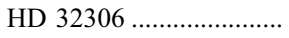 & 2 & 3 & 8 & 2.5 & 0.80 & $\ldots$ & $0 / 0$ & $\ldots$ & $\ldots$ & $\ldots$ & $\ldots$ \\
\hline 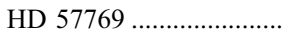 & 2 & 4 & 18 & 4.4 & 0.45 & $\ldots$ & $\ldots$ & $\ldots$ & $\ldots$ & $\ldots$ & $\ldots$ \\
\hline 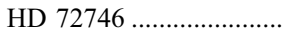 & 2 & 3 & 4 & 1.2 & 1.62 & $2 / 3$ on 1998 Dec 27 & $0 / 0$ & 3.0 & 1 & 2 & 9 \\
\hline 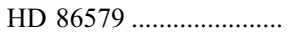 & 2 & 4 & 8 & 2.6 & 0.75 & $\ldots$ & $0 / 1$ & $\ldots$ & $\ldots$ & $\ldots$ & $\ldots$ \\
\hline 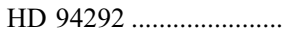 & 2 & 8 & 26 & 5.4 & 0.37 & $\ldots$ & $1 / 5$ & $\ldots$ & $\ldots$ & $\ldots$ & $\ldots$ \\
\hline$\xi \mathrm{UMa}$ & 2 & 7 & 58 & 10.6 & 0.20 & $\ldots$ & $\cdots$ & $\ldots$ & $\ldots$ & $\ldots$ & $\ldots$ \\
\hline HD $18884 \ldots \ldots \ldots \ldots \ldots \ldots \ldots$ & 2 & 5 & 3 & 0.1 & 14.49 & $\cdots$ & $\cdots$ & $\cdots$ & $\cdots$ & $\cdots$ & $\cdots$ \\
\hline 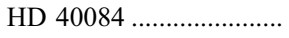 & 2 & 4 & 13 & 3.1 & 0.64 & $\ldots$ & $0 / 0$ & $\ldots$ & $\ldots$ & $\ldots$ & $\ldots$ \\
\hline 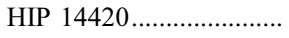 & 2 & 2 & 1 & 0.1 & 14.97 & $2 / 2$ on 2000 Dec 27 & $\ldots$ & 2.5 & 1 & 2 & 10 \\
\hline RT Lac ........................... & 2 & 7 & 42 & 12.7 & 0.16 & $\ldots$ & $0 / 1$ & $\cdots$ & $\cdots$ & $\cdots$ & $\cdots$ \\
\hline 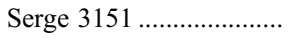 & 2 & 3 & 3 & 0.9 & 2.26 & $\ldots$ & $1 / 1$ & $\cdots$ & $\ldots$ & $\ldots$ & $\ldots$ \\
\hline 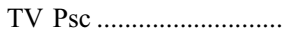 & 2 & 4 & 38 & 2.0 & 1.01 & $\ldots$ & $0 / 0$ & $\ldots$ & $\ldots$ & $\ldots$ & $\ldots$ \\
\hline 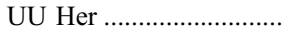 & 2 & 7 & 51 & 10.5 & 0.19 & $\ldots$ & $1 / 2$ & $\ldots$ & $\ldots$ & $\ldots$ & $\ldots$ \\
\hline
\end{tabular}

Notes.-Objects with two or more events in $\mathrm{DS}_{\text {clean }}$ listed with their observing statistics (number of events, triggers, observations, hours of cumulative observation, event rate in $\mathrm{hr}^{-1}$ ), nights with multiple events/triggers from one object (a dash indicates all events occurred on separate nights), and the number of events/triggers that were vetoed (not observed) by Princeton during concurrent observations (a dash indicates no concurrent observations in $\mathrm{DS}_{\text {clean }}$ ). Statistics from the Harvard instrument for joint Harvard/Princeton reobservations are listed in the four rightmost columns (which occurred after November 2003 and are not in $\mathrm{DS}_{\text {all }}$; a dash indicates no reobservations). No simultaneous Harvard/Princeton triggers were recorded during the reobservations. Table 6 lists coordinates and descriptions for these objects.

the idea that the two stars are a physical pair in orbit (for which their separation would be $\geq 17.6 \mathrm{AU}$ ).

\subsubsection{Other Objects}

No other object in Tables 5 and 6 displayed the extrastatistical properties of HD 220077. Several objects had long total observation times and are probably explained as statistical fluctuations in a constant background of Poisson-distributed events.

The six objects with multiple events in one night were deemed the most interesting and were reobserved for 1-3 hr. Although some events and triggers were recorded at Harvard during the joint reobservations, none of them were simultaneously seen at Princeton.

Of note in the reobserved group is DU Leo, which recorded three events and seven triggers in 40 minutes over two observations on 1999 May 30. Although this night was included in $\mathrm{DS}_{\text {clean }}$, it is worth noting that the 1999 season of corona discharge, with its attendant high event and trigger rates (Fig. 3), had begun a few days earlier. We find no set of triggers from DU Leo (or any other object) that passes the Rational Period test. Taking $r_{1}=0.075$ minute $^{-1}$ and $r_{2}=$ 0.006 minute $^{-1}$, the reobservation probabilities for this object are $p_{r_{1}} \sim 10^{-4}$ and $p_{r_{2}} \sim 0.5$.
The properties of many of the objects in Tables 5 and 6 (supergiants, multiple systems) make them less likely sites for Earth-like life. None of them are members of the Habitable Catalog (Turnbull \& Tarter 2003), a catalog of $\sim 17,000$ potentially habitable stars from the $\sim 120,000$ star Hipparcos catalog (Perryman 1997).

\subsubsection{Conclusions from Harvard Observations}

Based on the Harvard observations and reobservations, our conclusion is this: given the low event rates and corresponding small-number statistics, we have found no evidence of clustering or periodicity from any candidate star, and the events are distributed impartially among the targets. There is additionally no correlation of event rate with stellar magnitude, confirming the conclusion that Poisson doubly coincident "accidentals" do not contribute candidate events at ordinary single-photon count rates. Reobservations of those objects with multiple events in one night did not reveal sources of optical flashes. From the results so far, therefore, we conclude that we have found no evidence for pulsed optical beacons from extraterrestrial civilizations.

In considering this conclusion, one must keep in mind the possibility that a transmitting civilization might choose to send a solitary pulse, or, equivalently for our observational 
TABLE 6

Interesting Objects in $\mathrm{DS}_{\text {clean }}$-Astronomical Information

\begin{tabular}{|c|c|c|c|c|c|c|}
\hline Name & Other Name & R.A. & Decl. & Visual Mag. & $\begin{array}{c}\text { Parallax } \\
\text { (mas) }\end{array}$ & Description \\
\hline HD $14535 \ldots \ldots \ldots \ldots \ldots \ldots \ldots \ldots \ldots$ & HIP 11098 & 22253 & +571443 & 8 & $0.37(0.88)$ & A2 Ia-supergiant, variable \\
\hline 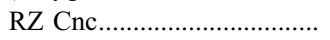 & HD 73343 & 83909 & +314744 & 9 & $3.25(1.56)$ & cool Algol binary, 21 day period \\
\hline 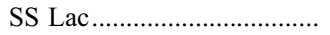 & HIP 108981 & 220442 & +462538 & 10 & $1.13(1.39)$ & triple, 14.4 and 678 day periods \\
\hline 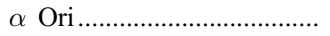 & HD 39801 & 55510 & +72425 & 1 & $7.73(1.64)$ & M2 Ib—supergiant, variable \\
\hline DU Leo & HD 84207 & 94411 & +252111 & 10 & - & G0 V-binary, 1.37 day period \\
\hline 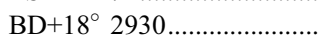 & GSC 03598-00615 & 144657 & +181800 & 9 & - & G8 V \\
\hline M67 1221................................ & GSC 01481-00366 & 85144 & +114426 & 11 & - & K1 III-binary, 6394 day period \\
\hline 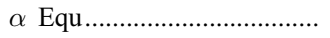 & HD 202448 & 211549 & +51452 & 4 & $17.51(0.89)$ & G0 III and A-binary, 99 day period \\
\hline $\mathrm{BD}+61^{\circ} 1045 \ldots \ldots \ldots \ldots \ldots \ldots \ldots$ & HD 70050 & 82316 & +612738 & 8 & $3.88(0.95)$ & G2 V-binary, 14.35 day period \\
\hline 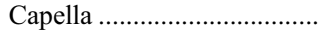 & HD 34029 & 51641 & +455953 & 0 & $77.29(0.89)$ & G5 III—binary, 104 day period \\
\hline 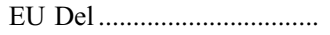 & HD 196610 & 203755 & +181607 & 6 & $9.16(0.99)$ & M6 III—semiregular variable \\
\hline 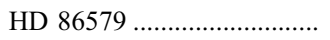 & HIP 48963 & 95919 & -30430 & 7 & $7.57(1.02)$ & F5 V-binary, 2729 day period \\
\hline 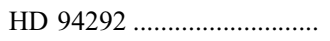 & HD 53212 & 105302 & +45743 & 8 & $13.02(0.92)$ & G5 V-double-lined binary \\
\hline$\xi \mathrm{UMa}$ & HD 98230/98231 & 111811 & +313145 & 4 & - & $\mathrm{F}$ and $\mathrm{G}$ dwarf-quadruple \\
\hline 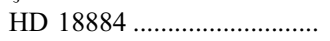 & HIP 14135 & 30217 & +40523 & 3 & $14.82(0.83)$ & M2 III-low-level variable \\
\hline 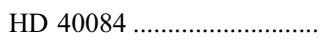 & HIP 28343 & 55922 & +495528 & 6 & $2.90(0.79)$ & G5 III—binary, 219 day period \\
\hline 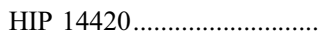 & HD 232747 & 30611 & +510606 & 10 & $14.64(1.79)$ & $\mathrm{K} 0 \mathrm{~V}$ \\
\hline RT Lac & HD 209318 & 220131 & +435326 & 9 & $5.19(1.05)$ & RS CVn binary, 5 day period \\
\hline Serge $3151 \ldots \ldots \ldots \ldots \ldots \ldots \ldots$ & GSC 03598-00615 & 212352 & +490737 & 11 & - & G0 V-spectroscopic binary \\
\hline TV Psc & HD 2411 & 02803 & +175335 & 5 & $6.65(0.78)$ & M3 III-semiregular variable \\
\hline UU Her ................................. & HIP 81272 & 163557 & +375802 & 8 & $-0.15(0.91)$ & $\mathrm{F} 2 \mathrm{Ib}$ - semiregular variable \\
\hline
\end{tabular}

Notes.-Right ascension (in hours, minutes, seconds; J2000.0), declination (in degrees, minutes, seconds), visual magnitude, parallax (mas, with uncertainties in brackets), and spectral types for the objects in Table 5. Information was not available where dashes are present.

protocol, a pulse repetition rate less than, say, once per hour. To put it another way, what do you do with isolated nonrepeating events - particularly when any one of them, if authentic, would constitute the greatest discovery in the history of humankind? You find a better way to do the experiment. It was this motivation that led to the construction of the Princeton experiment in 2001, and to the two years of joint observations, which are discussed below.

\subsection{Synchronized Observations with Princeton}

Given our current background level of roughly one event per night of observation with the Harvard instrument, a single optical pulse from an extraterrestrial civilization would likely be dismissed as a background event. To attract attention, the signal would have to consist of a succession of pulses from a source candidate, perhaps exhibiting nonrandom arrival times. As we remarked above, we recognize that this is a shortcoming of the experiment-we may miss a true pulsed beacon.

To address this problem, we duplicated the detector system at the $0.9 \mathrm{~m}$ Cassegrain at the Fitz-Randolph Observatory in Princeton, New Jersey. Since 2001 November, this telescope has followed the Harvard telescope through its nightly observing programs, synchronized via the internet. Given that the baseline between observatories is $L / c=T \approx 1.6 \mathrm{~ms}$ of light-time, the absolute timing precision of $0.1 \mu$ s permits us not only to identify approximate coincidences; it further defines an error band in the sky whose width is of order $\Delta \theta \approx \Delta \tau / T=12^{\prime \prime}$. This is comparable to the observed target field, set by the focal plane aperture stop. Thus, with good accuracy we can verify that a candidate two-observatory coincident event is consistent with the observing geometry.

To see how effective such a scheme is in eliminating uncorrelated events at the two observatories, imagine an event rate $r_{e}=1 \mathrm{hr}^{-1}$ at each observatory and let us require that each candidate event pair (between the two observatories) be within a broad time window of, say, $\Delta T=1 \mathrm{~ms}$ to be considered a confirmed detection (recall that our GPS-derived timing accuracy is in fact 4 orders of magnitude better). Then the combined background rate due to "pileup" is $r_{\text {both }}=$ $r_{e}^{2} \Delta T=3 \times 10^{-7}$ events $\mathrm{hr}^{-1}$, or one event every 3 million observing hours. With such a low background rate, we would have to examine seriously the astrophysical and extraterrestrial significance of even a single coincidence at the two observatories.

While the Princeton observatory provides excellent positive confirmation (simultaneous events would be believed with high confidence), we have less confidence in negative confirmation (vetos), particularly of low-amplitude events. Sensitivity varies at the two sites due to nonuniform photodetector gain, electronic gain, and environmental factors (e.g., thin clouds at one observatory). These caveats apply most strongly to low-amplitude signals; large pulses observed at Harvard should also be seen at Princeton. ${ }^{17}$

\footnotetext{
17 The effect of deadtime following corona-induced triggers is insignificanteven under poor conditions ( $\sim 50$ corona triggers per hour) the probability of missing a confirming event is $\lesssim 10^{-3}$.
} 


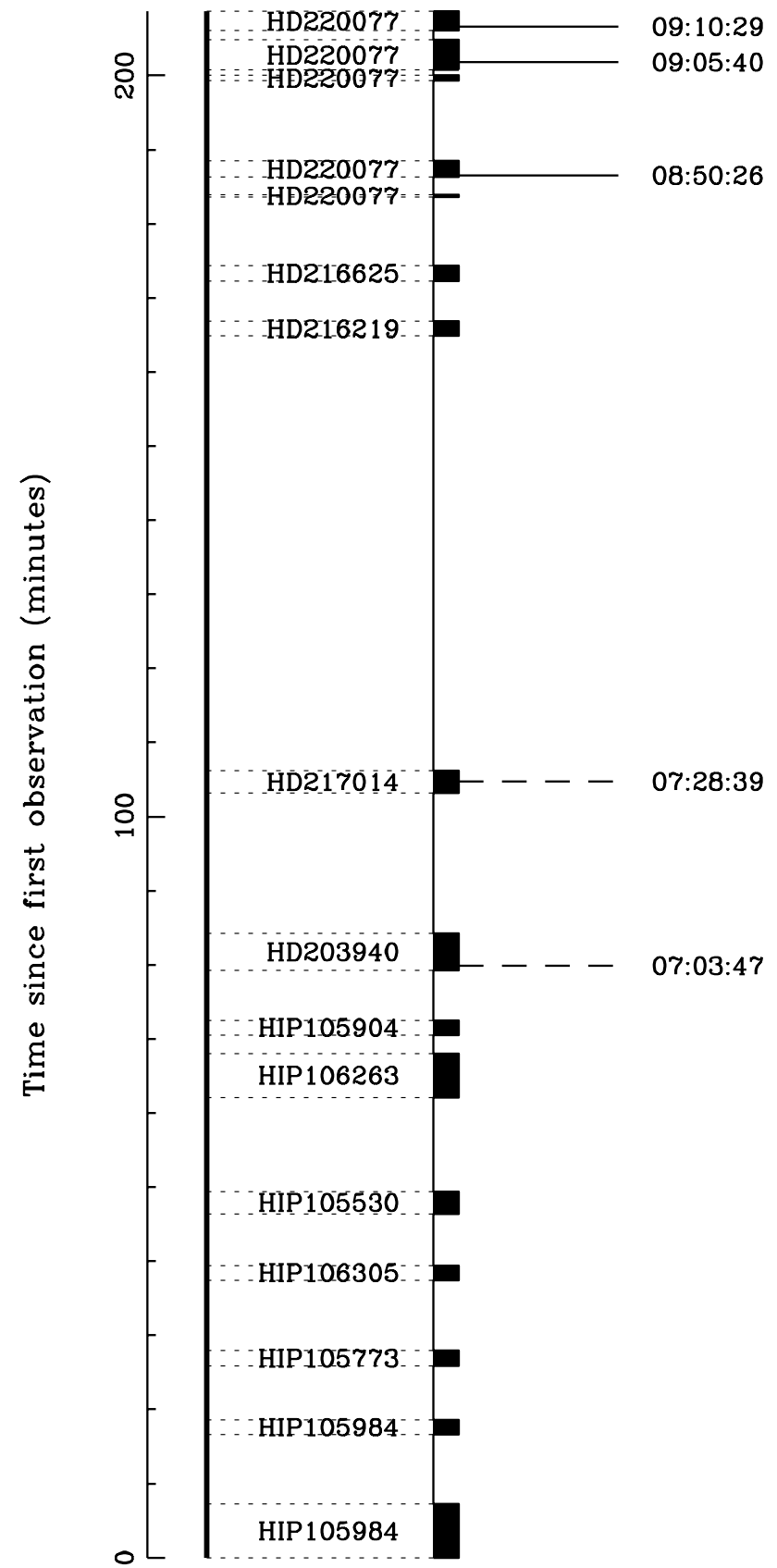

FIG. 6.-Observations on the night of 2000 November 4 beginning at 05:43:53 UT at Harvard. The boxes indicate observation intervals of objects listed below. Events (solid lines) and nonevent triggers (dashed lines) are shown above the observations with timestamps labeled. Note that all three events occurred during observations of HD 220077; two triggers that did not meet event criteria were recorded on other objects (HD 203940 and HD 217014). All observations are in $\mathrm{DS}_{\text {clean }}$, but not in $\mathrm{DS}_{\text {overlap. (These obser- }}$ vations are atypically short and sparse; see Fig. 7 for a typical night's observations.)

Table 1 summarizes the Princeton observations that coincided with 1721 Harvard observations of 1142 objects totaling $244 \mathrm{hr}$ of observation ( $\left.\mathrm{DS}_{\text {overlap }}\right)$. During these observations, Princeton recorded 2327 triggers and 429 events, while Harvard recorded 614 triggers and 130 events (106 triggers and 17 events in $\left.\mathrm{DS}_{\text {clean }}\right)$. The somewhat lower signal thresholds on the Princeton instrument, as well as higher corona rates, may account for the higher trigger and event rates at Princeton.

\subsubsection{HIP 107395}

During synchronized observations from 2001 November 17 onward, only one pair of triggers was recorded with arrival times that are consistent with an extraterrestrial or astrophysical optical pulse arriving at the geographically separated observatories. On 2003 September 17, during a joint observation of HIP 107395, Harvard recorded a trigger at 06:52:16.944 UT and Princeton recorded one at 06:52:16.943 UT (computer clock times). Unfortunately, the GPS clock at Princeton (with $0.1 \mu$ s accuracy) was not working for a few months around this date. Thus, unambiguous identification of an astrophysical or extraterrestrial pulse from HIP 107395 is not possible. Since the computer clock times are only accurate to $\sim 50 \mathrm{~ms}$, there is roughly a $2 \%$ chance that the triggers actually occurred within $\pm 1 \mathrm{~ms}$ of each other; the millisecond alignment may have been the work of chance. Although there are several other reasons to dismiss these as background triggers serendipitously recorded $1 \mathrm{~ms}$ apart (described below), it is worth noting that this is the only trigger pair whose arrival times are consistent with a single pulse arriving at both observatories. No other trigger pair arrived with a time separation of less than $0.3 \mathrm{~s}$.

As shown in Figure 7, the trigger rates at Harvard and Princeton were both elevated on 2003 September 17. Harvard recorded 23 triggers and two events during 32 observations of 25 objects over 6.1 observing hours that night, while Princeton recorded 315 triggers and 64 events during 15 observations of 15 objects over 3.5 observing hours. ${ }^{18}$ During the 20 minute observation of HIP 107395, Harvard recorded five triggers and zero events. Princeton observed HIP 107395 for 26.4 minutes (completely covering the Harvard observation) and recorded 32 triggers and 10 events (eight triggers occurred before Harvard was observing).

The probability of any pair of triggers randomly occurring within $\pm 1 \mathrm{~ms}$ during the observation of HIP 107395 can be estimated as $p_{1 \mathrm{~ms}}=r_{H} r_{P} \tau_{\text {coinc }} T_{\text {obsv }}=3 \times 10^{-5}$, where $r_{H}=$ $15 \mathrm{hr}^{-1}$ and $r_{P}=72 \mathrm{hr}^{-1}$ are the trigger rates at Harvard and Princeton during the $T_{\text {obsv }}=20$ minute observation, and $\tau_{\text {coinc }}=1 \mathrm{~ms}$ is the coincidence window. Of the 1123 obser-

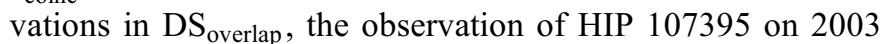
September 17 has the fifth largest $p_{1 \mathrm{~ms}}$ (because of high $r_{H}$, $\left.r_{P}\right)$. The probabilities from all observations can be combined to give the probability of one or more of those observations having a pair of triggers with in $\pm 1 \mathrm{~ms}$ : $p_{\text {all }}=1-\prod(1-$ $\left.p_{1 \mathrm{~ms}}\right)=2 \times 10^{-3}$, where the product is over the observations in $\mathrm{DS}_{\text {overlap. }}$

We considered the possibility that the inaccuracies in the Harvard and Princeton computer clocks were temporally correlated, for example, because the computer clocks are disciplined by identical computer programs and GPS clocks. A comparison of these inaccuracies during normal GPS functioning for Harvard and Princeton events that occurred even within 1 minute of each other revealed no such correlation.

It is worth noting that not only was this night's data excluded from $\mathrm{DS}_{\text {clean }}$, but furthermore that neither trigger qualified as an event. The Harvard trigger failed because the pulse recorded in one detector was $4 \mathrm{~ns}$ long, while the pulse in the other detector was longer than the MTD-135 observation window of 300 ns. The Princeton trigger failed because of an amplitude

\footnotetext{
18 These anomalously high rates are consistent with corona discharge; all observations on 2003 September 17 from Harvard were thus excluded from $\mathrm{DS}_{\text {clean }}$ by the algorithm described in $\S 4.1 .2$.
} 
TABLE 7

ObSERVATIONS OF HD 220077

\begin{tabular}{|c|c|c|c|c|c|c|c|c|c|c|c|c|}
\hline \multirow[b]{2}{*}{ Date } & \multirow[b]{2}{*}{$\mathrm{DS}_{\mathrm{all}}$} & \multirow[b]{2}{*}{$\mathrm{DS}_{\text {clean }}$} & \multirow[b]{2}{*}{$\mathrm{DS}_{\text {overlap }}$} & \multicolumn{4}{|c|}{ HD 220077} & \multicolumn{5}{|c|}{ Other OBJects } \\
\hline & & & & Observations & Minutes & Events & Triggers & Objects & Observations & Minutes & Events & Triggers \\
\hline 2000 Nov 4 ........... & yes & yes & $\ldots$ & 5 & 10 & 3 & 3 & 10 & 11 & 36 & 0 & 2 \\
\hline 2000 Nov $18 \ldots \ldots . .$. & yes & yes & $\ldots$ & 2 & 6 & 0 & 0 & 44 & 49 & 358 & 2 & 3 \\
\hline 2001 Jan $26 \ldots \ldots \ldots . . .$. & yes & yes & $\ldots$ & 1 & 2 & 0 & 0 & 52 & 57 & 399 & 0 & 0 \\
\hline 2002 Jul 7 .............. & yes & $\ldots$ & $\ldots$ & 1 & 4 & 0 & 0 & 19 & 23 & 255 & 2 & 14 \\
\hline 2002 Sep $13 \ldots \ldots \ldots . .$. & yes & $\ldots$ & $\ldots$ & 1 & 3 & 0 & 0 & 33 & 34 & 365 & 2 & 10 \\
\hline 2002 Oct $21 \ldots \ldots \ldots$ & yes & $\ldots$ & $\ldots$ & 1 & 4 & 0 & 0 & 45 & 51 & 410 & 1 & 11 \\
\hline 2003 Jan $15 \ldots \ldots \ldots . .$. & yes & yes & $\ldots$ & 1 & 3 & 0 & 0 & 22 & 29 & 329 & 0 & 0 \\
\hline 2003 Jul $16 \ldots \ldots \ldots . .$. & yes & $\ldots$ & $\ldots$ & 1 & 4 & 1 & 1 & 19 & 23 & 284 & 5 & 17 \\
\hline 2003 Aug $21 \ldots \ldots . .$. & yes & $\ldots$ & yes & 1 & 8 & 0 & 0 & 28 & 29 & 205 & 5 & 27 \\
\hline 2003 Sep $30 \ldots \ldots \ldots . .$. & yes & $\ldots$ & $\ldots$ & 1 & 8 & 0 & 0 & 29 & 37 & 282 & 6 & 14 \\
\hline 2004 Feb $12 \ldots \ldots \ldots . .$. & - & - & - & 4 & 61 & 0 & 0 & 38 & 45 & 408 & 1 & 1 \\
\hline 2004 Feb $14 \ldots \ldots \ldots$ & - & - & - & 2 & 38 & 0 & 0 & 20 & 30 & 413 & 2 & 7 \\
\hline
\end{tabular}

Notes.-Observations of HD 220077. Columns detail the number of observations, the observation duration in minutes, and the number of events and triggers for HD 220077 and other objects observed that night. The data sets that each observation belongs to are labeled with "yes." Observations on 2004 February 12 and 14 were reobservations with Princeton after the closing date of the three listed data sets. Note that HD 220077 had three events, and no nonevent triggers, on 2000 November 4.

mismatch in the two detectors (first and fourth thresholds exceeded). These are both symptoms of corona discharge.

Although the trigger timings are consistent with an astrophysical or extraterrestrial optical pulse, we believe that random coincident background noise is a far more likely explanation. The lack of confirmatory GPS timing, the higher trigger rates on 2003 September 17, and the triggers' failure to meet event criteria all contribute to this conclusion. Extraordinary claims require extraordinary evidence, which is lacking here.

Nevertheless, even the remote possibility of a worldchanging discovery warrants significant reobservations. Over three nights (2003 November 25, 2003 December 2-3), we conducted seven joint reobservations of HIP 107395 (with GPS restored) for a total of 140 additional minutes. No triggers were recorded at Harvard, while Princeton recorded one trigger and no events on 2003 December 2.

The implications of this null confirmation depend on hypothetical source scenarios: if we assume a $50 \%$ probability of detecting a pulse during the initial 20 minute observation of HIP 107395 on 2003 September 17 (that is, a signal repetition rate of $1.5 \mathrm{hr}^{-1}$ ), then the probability of not detecting a pulse in the following seven 20 minute observations is $1 / 2^{7} \approx 10^{-2}$. If we assume (rather unrealistically) that we had a $50 \%$ probability of detecting a pulse from any object during the $162 \mathrm{hr}$ of joint observations (that is, a rate of $3.1 \times 10^{-3} \mathrm{hr}^{-1}$ ), then the probability of not detecting a pulse in the following seven 20 -minute observations is $\sim 0.993$. Thus, if the signal repeats often, the reobservations reinforce our belief that background noise caused the $1 \mathrm{~ms}$ trigger pair. If the beacon is broadcast infrequently, the reobservations tell us little.

HIP $107395 \quad$ (R.A. $=21^{\mathrm{h}} 45^{\mathrm{m}} 10^{\mathrm{s}} ; \quad$ decl. $=-0^{\circ} 30^{\prime} 30^{\prime \prime}$; J2000.0) is an 11th visual magnitude late $\mathrm{K}$ dwarf with a parallax of $17.55 \pm 2.85$ mas (implying a range of $\sim 60 \mathrm{pc}$ ). It is being surveyed for radial velocity companions for next generation microwave SETI. HIP 107395 is also a member of the Habitable Catalog (Turnbull \& Tarter 2003).

\subsection{Implications}

The foregoing results can be summarized as follows.

1.- The Harvard instrument made 15,897 observations of 6176 stars totaling $2378 \mathrm{hr}$. During these observations $\left(\mathrm{DS}_{\text {all }}\right)$ it detected 4746 triggers with a subset of 1117 events. When we remove the observations characterized by humidity-induced corona with $\left(\mathrm{DS}_{\text {clean }}\right)$, we are left with 11,600 observations of 4730 stars over $1721 \mathrm{hr}$, yielding 274 events and 1066 triggers. These events appear to be Poisson distributed in time and uncorrelated with the target's brightness and the observing geometry.

2.- The Princeton instrument has observed in tandem with the Harvard instrument for 1721 observations ( $\left.\mathrm{DS}_{\text {overlap }}\right)$ of 1142 objects totaling $244 \mathrm{hr}$. The arrival times for one HarvardPrinceton trigger pair are consistent with receiving an optical pulse at the geographically separated observatories (within the accuracy of the computer clocks). For multiple reasons, we believe these triggers resulted from background noise sources.

\subsubsection{Scenarios}

The implications of our data depend on the model that they are testing. Of the possible intentional optical pulsed signals that an extraterrestrial civilization could generate, let us consider the implications of just two scenarios.

Scenario 1: A fraction $f$ of the stars in our region of the Galaxy harbor civilizations that transmit optical signals to Earth that our experiment could detect. The signal is composed of multiple pulses in fast succession (less than our minimum observation time of 2 minutes) displaying some hallmark of intelligence (e.g., nonrandom arrival times). This signal is broadcast repeatedly with a period $P$ (greater than our maximum observation time).

Scenario 2: A fraction $f$ of the stars in our region of the Galaxy harbor civilizations that transmit optical signals to Earth that our experiment could detect. The signal is composed of a single pulse that is broadcast repeatedly with a period $P$ (greater than our maximum observation time).

While these scenarios might be considered simplistic, the implications for our data are nonetheless instructive. The Harvard search, with its sensitivity to multiple pulses but not solitary pulses, is an excellent probe of scenario one. Scenario 2 requires a background rate of zero- a good match for the Harvard-Princeton search.

\subsubsection{Fraction of Stars with Transmitting Civilizations}

An upper bound on the fraction of stars in our region of the Galaxy that are signaling Earth with optical flashes can be 


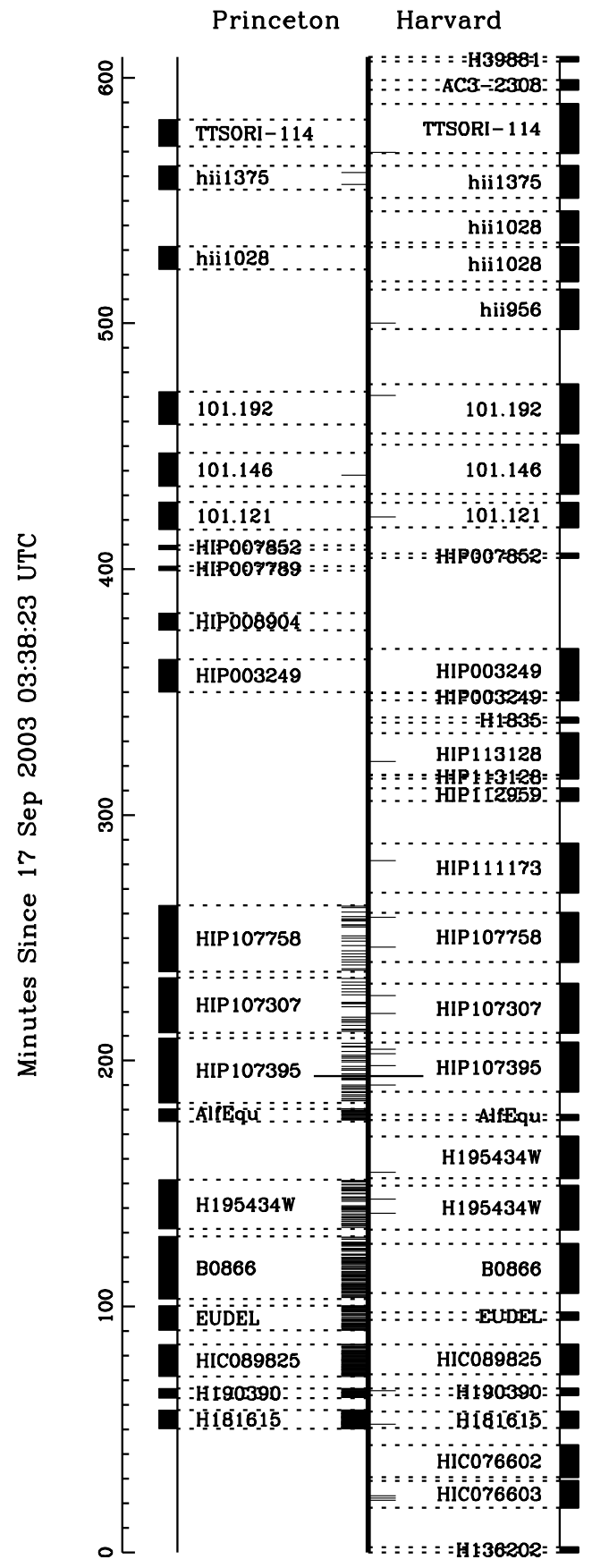

Fig. 7.-Observational diagram for the night of 2003 September 17. Observations at Harvard (right) and Princeton (left), with time increasing upward. Observations are designated by solid boxes and dashed lines to the central vertical axis. Object names are written between the dashed lines. Triggers are shown as short horizontal lines coming out of the central vertical axis (Harvard to the right, Princeton to the left). These trigger marks are extended for a pair of triggers whose arrival times at Harvard and Princeton were $1 \mathrm{~ms}$ apart, as recorded by the computer clocks (see $\S 4.3 .1$ ). Note that the trigger rates were elevated at both observatories, particularly during the first half of the night.

calculated as a function of $P$. In the analysis that follows, paralleling Horowitz \& Sagan (1993), we assume that none of the Harvard or Harvard-Princeton observations detected signals from extraterrestrial civilizations.

The probability of detecting an extraterrestrial signal during an observation of duration $t_{\text {obsv }}$ is $p_{\text {obsv }}(P)=\min \left(1, t_{\text {obsv }} / P\right)$,

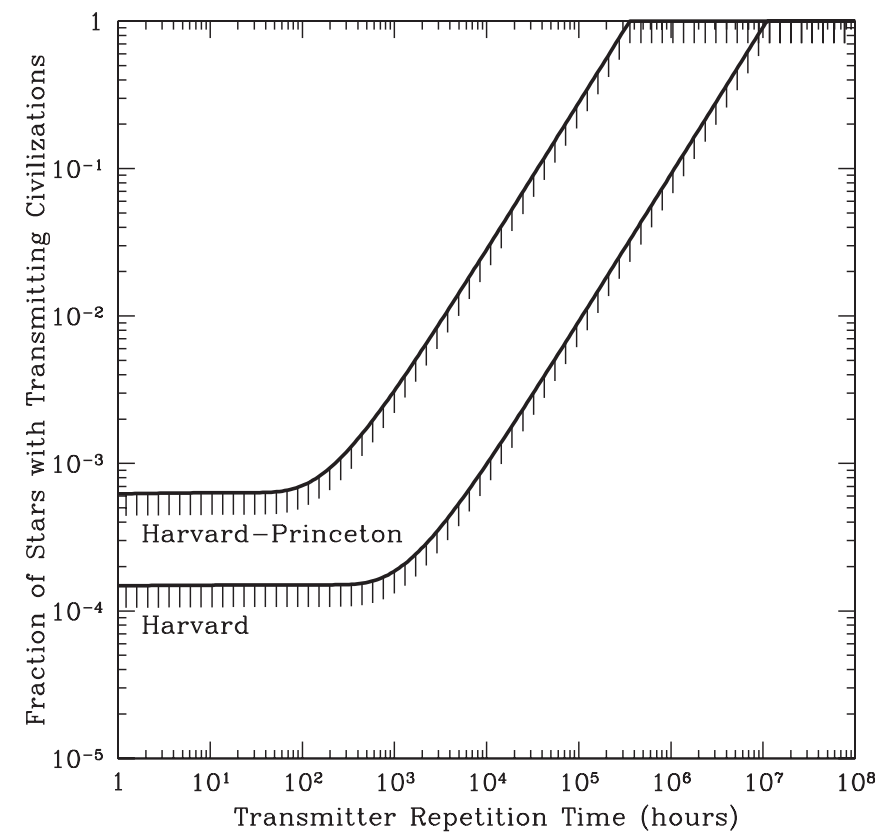

FIG. 8.-Upper bounds on the fraction of stars with transmitting civilizations as a function of transmitter repetition time for the Harvard experiment (scenario one; $\mathrm{DS}_{\text {clean }}$ ) and the Harvard-Princeton experiment (scenario two; $\mathrm{DS}_{\text {overlap }}$ ). Each curve asymptotes to $f=1 / N_{\mathrm{obj}}$ for small $P$ and cuts off at $f=1$ for $P \geq T / \ln 2$.

where the minimum function bounds $p_{\text {obsv }}(P) \leq 1$. The probability of detecting a signal from any one object is:

$$
p_{\text {obj }}(P)=1-\prod_{\text {obsv }}\left[1-p_{\text {obsv }}(P)\right] .
$$

The expected number of signal detections during the entire program, $S$, is the sum of the objects' probabilities times the fraction of objects that are transmitting: $S=f \sum_{\mathrm{obj}} p_{\mathrm{obj}}$. We adjust $f$ so that the Poisson probability of observing zero extraterrestrial signals $\left(e^{-S}\right)$ is 0.5 ; that is, we choose $f$ so that the observing program has a $50 \%$ chance of success. Solving for $f$, we obtain an upper bound on the fraction of transmitting civilizations:

$$
f(P)=\min \left[1, \frac{\ln 2}{\sum_{\text {obj }} p_{\text {obj }}(P)}\right],
$$

where the minimum function limits stars to at most one transmitting civilization.

Figure 8 shows upper bounds on $f(P)$ for scenario 1 (Harvard; $\mathrm{DS}_{\text {clean }}$ ) and scenario 2 (Harvard-Princeton; $\left.\mathrm{DS}_{\text {overlap }}\right)$. The two limits of $P$ are of interest. For high repetition rate transmitters $(P \ll T), f \rightarrow 1 / N_{\text {obj. }}$. (The total observing time is $T=$ $\sum_{\text {obsv }} t_{\text {obsv. }}$ ) There is a cutoff repetition rate, $P \geq T / \ln 2$, above which $f=1$ and we cannot say anything about the density of transmitters. Also note that the Harvard limit is below and to the right of the Harvard-Princeton limit because the latter derives from fewer objects observed for less time.

\section{FUTURE DIRECTIONS}

\subsection{Lessons Learned}

Here we briefly list suggestions for the designers of future optical SETI instruments: 
A second observatory, coupled with precise event timing, completely eliminates background events. Adding the Princeton instrument allowed us to search for a class of signals that the Harvard instrument alone could not (solitary pulses), and provides for the strongest possible confirmation of a genuine signal.

Making one's instrument robust against many failure modes - reliable software, good telemetry (both environmental and electronic - e.g., temperature, humidity, power supply voltages), mechanical interlocks, and optical fibers for lightning protection-is key for continued semiautonomous use over several years.

The benefits of performing diagnostic checks before every observation cannot be overemphasized. In an experiment looking for rare events, it is essential to know the condition of the apparatus. Many of our investigations into the origins of events would have been impossible without diagnostic data.

We also recommend a complete end-to-end test, ideally with a source in the far field. Our instruments did nearly this - an LED test-flash, albeit at the beam splitter - before every observation.

\subsection{New Directions in Optical SETI}

Optical SETI is a nascent field with room to grow in many directions. Targeted searches of larger numbers of stars are important if the number of transmitting civilizations is not large. Targeted SETI can also benefit from better target selection using the wealth of astrophysical data available now and in the near term.

Pulsed infrared SETI has the distinct advantages (from the transmitter's perspective) of greater signaling range (as a result of decreased scattering and absorption in the IR), lower energy cost per photon, and decreased stellar background. However, contemporary detector technology requires cryogenics and is often limited by small sensitive area and slow response. Major improvements in detector technology could enable pulsed IR searches.

Targeted searches have a significant shortcoming -in our case, for example, five years of data collection has covered barely $10^{-5}$ of the sky area. With $\sim 10^{6}$ Sun-like stars within
1000 lt-yr, and the possibility that advanced life may exist in the voids between stars (Dyson 2003), a complementary observing strategy of targeted searches and sky surveys represents the greatest chance for success in optical SETI. For this purpose we are building a $1.8 \mathrm{~m} \mathrm{f} / 2.5$ wide-field telescope with a pixelated fast photodetector, to view (in transit mode) a $1.6 \times 0.2$ field with a pair of 512 pixel photodetectors; each pixel will be digitized at 1 Gsps, permitting accurate reconstruction of pulsed light profiles. ${ }^{19}$ It will scan the sky once in $1200 \mathrm{hr}$ - roughly 150 clear nights - with a minimum viewing time of 48 s per target. First light is expected in 2004-2005. For more detail, see Horowitz (2004) and Howard et al. (2004).

Optical SETI could benefit also from collaboration with a cosmic-ray or gamma-ray observatory (Covault 2001). Such instruments have large areas (tens of square meters) and sensitivity to nanosecond-scale optical pulses, but routinely discard pulses coming from point sources because such events are inconsistent with diffuse air showers.

The Harvard SETI group gratefully acknowledges the enlightened and continued support of the Planetary Society (TPS) and the Bosack-Kruger Charitable Foundation, and additional support from the SETI Institute. The Princeton SETI group thanks Eric Tilenius, Princeton University, and the Princeton University Physics Department for financial support. We are indebted to the night observers at Oak Ridge Observatory and at the Fitz-Randolph Observatory for their tireless efforts that bring us so many photons. We particularly thank Joe Caruso and Guillermo Torres at ORO, and Herman Ashley, Rhonda and Richard Gillingwater, Maggie Kirkland, Mark Lopez, Joseph Maffei, Paul Mark, John Miller, Aaron Schomburg, James Wray, and others (Willman 2004) at FRO. We thank also Chris Clearfield, Jason Gallicchio, Chris Laumann, Alan Sliski, Pratheev Sreetharan, and Anne Sung at Harvard.

\footnotetext{
19 The resultant data rate-3.5 Tbps - is equivalent to the contents of all books in print, every second.
}

Bhathal, R. 2001, in Proc. SPIE, 4273, 144

Cocconi, G., \& Morrison, P. 1959, Nature, 184, 844

Coldwell, C. 2002, Ph.D. thesis, Harvard Univ.

Cordes, J. M. 2002, SETI 2020 (1st ed.; Mountain View: SETI Press), 427

Cordes, J. M., \& Lazio, T. J. 1991, ApJ, 376, 123

Covault, C. E. 2001, in Proc. SPIE, 4273, 161

Danner, R., \& Unwin, S. 1999, SIM (Space Interferometry Mission): Taking the Measure of the Universe (Washington DC: NASA/JPL)

Dyson, F. J. 2003, Int. J. Astrobiol., 2, 103

Ekers, R., Cullers, K., Billingham, J., \& Scheffer, L., ed. 2002, SETI 2020 (1st ed.; Mountain View: SETI)

Horowitz, P. 2004, Harvard Optical SETI Web page, http://www.oseti.org

Horowitz, P., \& Sagan, C. 1993, ApJ, 415, 218

Howard, A. W., \& Horowitz, P. 2001, in Proc. SPIE 4273, The Search for Extraterrestrial Intelligence (SETI) in the Optical Spectrum III, ed. S. A. Kingsley \& R. Bhathal (Bellingham: SPIE), 153

Howard, A. W., Horowitz, P., Coldwell, C. M., Stefanik, R., Gallicchio, J., \& Laumann, C. 2004, in Proc. 54th IAF Congress, in press

Krupke, W. F. 1997, in Proc. SPIE, 3047, 73

\section{REFERENCES}

Lampton, M. 2000, in ASP Conf. Ser. 213, Bioastronomy 99, ed. G. A. Lemarchand \& K. J. Meech (San Francisco: ASP), 565

Latham, D. 2000, in ASP Conf. Ser. 213, Bioastronomy 99, ed. G. A. Lemarchand \& K. J. Meech (San Francisco: ASP), 137

Latham, D. W. 1992, in IAU Colloq. 135, Complementary Approaches to Double and Multiple Star Research, ed. H. A. McAlister \& W. I. Hartkopf (ASP Conf. Ser. 32; San Francisco: ASP), 110

Mathis, J. S. 1990, ARA\&A, 28, 37

Perryman, M. A. C., et al. 1997, A\&A, 323, L49

Reines, A. E., \& Marcy, G. W. 2002, PASP, 114, 416

Ross, M. 1965, Proc. IEEE, 53, 1780

Rossi, B. 1948, Rev. Mod. Phys., 20, 537

Schwartz, R., \& Townes, C. 1961, Nature, 190, 205

Townes, C. 1983, Proc. Natl. Acad. Sci., 80, 1147

Turnbull, M. C., \& Tarter, J. C. 2003, ApJS, 149, 423

Willman, A. 2004, Princeton Optical SETI Contributors, http://observatory. princeton.edu/oseti/contributors.html

Wright, S. A., Drake, F., Stone, R. P., Treffers, D., \& Werthimer, D. 2001, in Proc. SPIE, 4273,173 